\title{
Global properties of codimension two spacelike submanifolds in Minkowski space
}

\author{
Shyuichi Izumiya*, Juan José Nuño Ballesteros \\ and María del Carmen Romero Fuster ${ }^{\dagger}$ \\ (Communicated by K. Ono)
}

\begin{abstract}
We consider codimension two spacelike submanifolds with a parallel normal field (i.e. vanishing normal curvature) in Minkowski space. We use the analysis of their contacts with hyperplanes and hyperquadrics in order to get some global information on them. As a consequence we obtain new versions of Carathéodory's and Loewner's conjectures on spacelike surfaces in 4-dimensional Minkowski space and 4-flattenings theorems for closed spacelike curves in 3dimensional Minkowski space.
\end{abstract}

Key words. Lightlike principal curvature directions, lightlike principal asymptotic directions, lightlike umbilics, flattenings.

2000 Mathematics Subject Classification. 53C40, 53A35

\section{Introduction}

The study of the contacts of submanifolds with hyperplanes and hyperspheres (i.e., totally umbilical hypersurfaces) in Euclidean space by means of the analysis of the singularities of appropriate functions has been useful in order to obtain global results concerning their geometry and topology. For instance, a classical consequence of Morse Theory establishes that $a$ closed (compact without boundary) CW-complex is a 2-sphere if and only if it admits some Morse function with exactly two critical points. Also, from the Extrinsic Geometry viewpoint, there is the following result due to Nomizu and Rodriguez ([29]): Every distance squared Morse function on a closed connected Riemannian n-manifold $M$ in the Euclidean space has index 0 or $n$ at all of its critical points if and only if $M$ is embedded as a Euclidean n-sphere.

\footnotetext{
*Work partially supported by Grant-in-Aid for formation of COE "Mathematics of Nonlinear Structure via Singularities".

${ }^{\dagger}$ Work of second and third authors has been partially supported by DGCYT grant no. MTM2006-06027 and FEDER.
} 
On the other hand, the study of the degenerate contacts of curves with hyperplanes in Euclidean $n$-space has led to several results on the existence of flattenings (zeroes of the $(n-1)$ th curvature function) for closed curves with appropriate convexity conditions ([1], [32], [34], [37], [39]).

In the case of surfaces immersed in 3-space, there is a conjecture, classically known as the Carathéodory conjecture, that asserts that any 2-sphere immersed in $\mathbb{R}^{3}$ has at least two umbilical points (critical points of its principal configuration). Such points can also be characterized as corank 2 singularities of distance squared functions on the surfaces. A generic proof of this result is due to E. A. Feldman ([5]), who showed that generically immersed 2-spheres must have at least 4 of them. The general case remains as a conjecture so far. An attempt to prove it has led to the following.

Loewner's conjecture. The index of an umbilic point of any surface immersed in $\mathbb{R}^{3}$ is at most one.

Several works have been devoted to the proof of this conjecture in the real analytic case ([12], [23],[38]). More recently, V. V. Ivanov has given a more complete version in [14]. Some other works, intended to prove the conjecture in the smooth case, have been done by R. Garcia, C. Gutierrez, F. Mercuri and Sánchez-Bringas ([9], [10]) and by B. Smyth and F. Xavier ([35]).

A generalization of Feldman's result for convex surfaces generically immersed in Euclidean 4-space was obtained in [7] as a consequence of the study of the generic behaviour of height functions on them.

This paper is the sequel of a recently appeared work of the first and third authors [20], concerning the geometrical properties related to the contacts of codimension 2 spacelike submanifolds with lightlike hyperplanes in Minkowski space. It was there proven that an analog of the Gauss-Bonnet theorem holds in this context. There were obtained some consequences for the particular case of spacelike submanifolds with a parallel normal field. Our aim here consists in obtaining further global results for these submanifolds. For this purpose we use the following basic idea, which is based in the method introduced by the second author in [30] in order to obtain a proof for the Carathéodory conjecture for analytic surfaces with vanishing normal curvature in 4-dimensional Euclidean space. We show that the properties related to the contacts with hyperplanes of spacelike $(n-1)$ submanifolds with vanishing normal curvature in Minkowski $(n+1)$-space can be put in terms of the corresponding properties for hypersurfaces in hyperbolic $n$-space. And then, by means of the conformal map which is given by the composition of the stereographic projections, in terms of properties concerning the contacts of hypersurfaces with hyperspheres in Euclidean $n$-space. In this way, under appropriate assumptions, we can "transport" to the first known results on the last ones.

The paper is organized as follows: Sections $2-4$ contain the notation and some preliminary results concerning spacelike submanifolds of codimension 2 in Minkowski space. In Section 3, we consider the shape operators associated to different normal fields on the submanifolds together with their corresponding principal configurations. A spacelike submanifold of codimension 2 in Minkowski space has a well defined lightcone Gauss map whose associated shape operator is known as the normalized lightcone shape opera- 
tor [20], and the corresponding principal configuration is known as the lightcone principal configuration. In the particular case of hypersurfaces in hyperbolic space, these coincide respectively with the horospherical shape operator and the horospherical principal configuration [17]. This is discussed in Section 4. In Section 5 we consider three naturally defined families of height function, respectively called timelike, spacelike and lightlike. Associated to the degenerate singularities of such functions we have the concepts of osculating hyperplanes and principal asymptotic directions. We see here, that in the case hypersurfaces of hyperbolic space, their contacts with hyperspheres, equidistant hypersurfaces and hyperhorospheres can be described in terms of height functions. Then as a consequence of the characterization of metric spheres in hyperbolic space due to Cecil and Ryan in [4], we can assert:

a) Suppose that $M$ is a compact connected smooth ( $n-1)$-manifold immersed in hyperbolic n-space. Then every non-degenerate timelike height function has exactly two critical points if and only if $M$ is embedded as a metric $(n-1)$-sphere.

b) Suppose that $M$ is a connected complete smooth hypersurface in hyperbolic nspace. Then every non-degenerate timelike or spacelike height function on $M$ has index 0 or $(n-1)$ if and only if $M$ is embedded as a hypersphere, hyperhorosphere, or equidistant hypersurface.

In Section 6 we concentrate our attention to codimension 2 submanifolds with a parallel normal field and show that, analogously to what happens with codimension 2 submanifolds of Euclidean space, the following property, that shall be fundamental for the global results of Section 7, also holds for spacelike codimension 2 submanifolds in Minkowski space:

If $M$ admits some globally defined parallel vector field, then there exists an orthonormal frame of common eigenvectors for the shape operators associated to all normal fields over $M$.

Moreover, we show that provided $M$ admits a non-degenerate unit timelike normal field whose image $\bar{M}$ in hyperbolic $n$-space has no self-intersections (i.e. is embedded), $M$ and $\bar{M}$ have the "same kind of contacts" with hyperplanes.

This allows us to conclude that, although in the general case of a spacelike $(n-1)$ submanifold of Minkowski $(n+1)$-space we cannot ensure the existence of any principal asymptotic direction at every point, those admitting a parallel normal field have exactly $(n-1)$ orthogonal principal asymptotic directions at every (non-critical) point.

Finally, in Section 7 we use the above properties in order to transport known global results concerning contacts of hypersurfaces with hyperspheres in Euclidean space to new results concerning the flat geometry of spacelike codimension 2 submanifolds with hyperplanes in Minkowski space, such as:

1. Suppose that $M$ is a compact connected smooth $(n-1)$-manifold immersed in Minkowski $(n+1)$-space. Then $M$ is a metric $(n-1)$-sphere contained in a spacelike hyperplane if and only if $M$ has a globally defined non-degenerate parallel normal field and every non-degenerate timelike height function has exactly two critical points on M.

2. Loewner's and Carathéodory's conjectures on umbilic points of surfaces in Euclidean 3-space hold if and only if they hold for lightcone umbilics of semiumbilical spacelike surfaces in Minkowski 4-space. So, relying on the analytic version of Loewner's conjecture for surfaces in Euclidean 3-space ([14]), we can assert that analytic semi- 
umbilical spacelike 2-sphere immersed in Minkowski 4-space have at least two lightcone umbilics.

3. Any closed curve that admits a globally defined non-degenerated parallel timelike normal field $\nu$ has at least two flattening points. If $\nu$ satisfies that $\nu(s) \neq \nu\left(s^{\prime}\right)$, for $s \neq s^{\prime}$, then the curve has at least 4 flattening points.

This leads to the following 4-vertex theorems for closed spacelike curves in the de Sitter 2-space and the 2-dimensional lightcone:

3a) Any regular closed spacelike curve immersed in de Sitter 2-space with nonvanishing curvature and geodesic curvature functions has at least 4 geodesic vertices (flattening points).

3b) Any regular closed spacelike curve immersed in the 2-dimensional lightcone with non-vanishing Gauss curvature function has at least 4 flattening points.

\section{Basic facts and notations on Minkowski space}

We introduce in this section some basic notations on Minkowski $(n+1)$-space and spacelike submanifolds. For basic concepts and properties, see [31].

Let $\mathbb{R}^{n+1}=\left\{\left(x_{0}, x_{1}, \ldots, x_{n}\right) \mid x_{i} \in \mathbb{R}(i=0,1, \ldots, n)\right\}$ be an $(n+1)$-dimensional cartesian space. For any $\mathbf{x}=\left(x_{0}, x_{1}, \ldots, x_{n}\right), \mathbf{y}=\left(y_{0}, y_{1}, \ldots, y_{n}\right) \in \mathbb{R}^{n+1}$, the pseudo scalar product of $\mathbf{x}$ and $\mathbf{y}$ is defined by

$$
\langle\mathbf{x}, \mathbf{y}\rangle=-x_{0} y_{0}+\sum_{i=1}^{n} x_{i} y_{i}
$$

We call $\left(\mathbb{R}^{n+1},\langle\rangle,\right)$ Minkowski $(n+1)$-space. We denote $\mathbb{R}_{1}^{n+1}$ instead of $\left(\mathbb{R}^{n+1},\langle\rangle,\right)$. We say that a non-zero vector $\mathbf{x} \in \mathbb{R}_{1}^{n+1}$ is spacelike, lightlike or timelike if $\langle\mathbf{x}, \mathbf{x}\rangle>0$, $\langle\mathbf{x}, \mathbf{x}\rangle=0$ or $\langle\mathbf{x}, \mathbf{x}\rangle<0$ respectively. The norm of the vector $\mathbf{x} \in \mathbb{R}_{1}^{n+1}$ is defined by $\|\mathbf{x}\|=\sqrt{|\langle\mathbf{x}, \mathbf{x}\rangle|}$. We have the canonical projection $\pi: \mathbb{R}_{1}^{n+1} \longrightarrow \mathbb{R}^{n}$ defined by $\pi\left(x_{0}, x_{1}, \ldots, x_{n}\right)=\left(x_{1}, \ldots, x_{n}\right)$. Here we identify $\{\mathbf{0}\} \times \mathbb{R}^{n}$ with $\mathbb{R}^{n}$ and it is considered as Euclidean $n$-space whose scalar product is induced from the pseudo scalar product $\langle$,$\rangle . For a vector \mathbf{v} \in \mathbb{R}_{1}^{n+1}$ and a real number $c$, we define a hyperplane with pseudo normal $\mathrm{v}$ by

$$
H P(\mathbf{v}, c)=\left\{\mathbf{x} \in \mathbb{R}_{1}^{n+1} \mid\langle\mathbf{x}, \mathbf{v}\rangle=c\right\} .
$$

We call $H P(\mathbf{v}, c)$ a spacelike hyperplane, a timelike hyperplane or a lightlike hyperplane if $\mathbf{v}$ is timelike, spacelike or lightlike respectively.

The hyperbolic $n$-space is given by

$$
H_{+}^{n}(-1)=\left\{\mathbf{x} \in \mathbb{R}_{1}^{n+1} \mid\langle\mathbf{x}, \mathbf{x}\rangle=-1, x_{0}>0\right\} .
$$

Any non-empty hypersurface of $H_{+}^{n}(-1)$ determined by the intersection of $H_{+}^{n}(-1)$ with either a spacelike, a timelike or a lightlike hyperplane is respectively called hypersphere, equidistant hyperplane or hyperhorosphere. 
Other well-known pseudo-spheres in Minkowski space are the de Sitter n-space, given by

$$
S_{1}^{n}=\left\{\mathbf{x} \in \mathbb{R}_{1}^{n+1} \mid\langle\mathbf{x}, \mathbf{x}\rangle=1\right\} .
$$

And the (open) lightcone:

$$
L C^{*}=\left\{\mathbf{x}=\left(x_{0}, x_{1}, \ldots, x_{n}\right) \in \mathbb{R}_{1}^{n+1} \mid x_{0} \neq 0,\langle\mathbf{x}, \mathbf{x}\rangle=0\right\} .
$$

The subset

$$
L C_{+}^{*}=\left\{\mathbf{x} \in L C^{*} \mid x_{0}>0\right\}
$$

is called future lightcone. We denote the $n$-dimensional lightcone with vertex $\lambda \in \mathbb{R}_{1}^{n+1}$ by

$$
L C_{\lambda}^{*}=\left\{\mathbf{x} \in \mathbb{R}_{1}^{n+1} \mid\langle\mathbf{x}-\lambda, \mathbf{x}-\lambda\rangle=0\right\} .
$$

If $\mathbf{x}=\left(x_{0}, x_{1}, \ldots, x_{2}\right)$ is a non-zero lightlike vector, then $x_{0} \neq 0$ and we define

$$
\widetilde{\mathbf{x}}=\left(1, \frac{x_{1}}{x_{0}}, \ldots, \frac{x_{2}}{x_{0}}\right) \in S_{+}^{n-1}=\left\{\mathbf{x}=\left(x_{0}, x_{1}, \ldots, x_{n}\right) \mid\langle\mathbf{x}, \mathbf{x}\rangle=0, x_{0}=1\right\} .
$$

We call $S_{+}^{n-1}$ the lightcone (or, spacelike) unit $(n-1)$-sphere.

For any $\mathbf{x}_{1}, \mathbf{x}_{2}, \ldots, \mathbf{x}_{n} \in \mathbb{R}_{1}^{n+1}$, we define a vector $\mathbf{x}_{1} \wedge \mathbf{x}_{2} \wedge \cdots \wedge \mathbf{x}_{n}$ by

$$
\mathbf{x}_{1} \wedge \mathbf{x}_{2} \wedge \cdots \wedge \mathbf{x}_{n}=\left|\begin{array}{cccc}
-\mathbf{e}_{0} & \mathbf{e}_{1} & \cdots & \mathbf{e}_{n} \\
x_{0}^{1} & x_{1}^{1} & \cdots & x_{n}^{1} \\
x_{0}^{2} & x_{1}^{2} & \cdots & x_{n}^{2} \\
\vdots & \vdots & \ddots & \vdots \\
x_{0}^{n} & x_{1}^{n} & \cdots & x_{n}^{n}
\end{array}\right|
$$

where $\mathbf{e}_{0}, \mathbf{e}_{1}, \ldots, \mathbf{e}_{n}$ is the canonical basis of $\mathbb{R}_{1}^{n+1}$ and $\mathbf{x}_{i}=\left(x_{0}^{i}, x_{1}^{i}, \ldots, x_{n}^{i}\right)$. We can easily check that

$$
\left\langle\mathbf{x}, \mathbf{x}_{1} \wedge \mathbf{x}_{2} \wedge \cdots \wedge \mathbf{x}_{n}\right\rangle=\operatorname{det}\left(\mathbf{x}, \mathbf{x}_{1}, \ldots, \mathbf{x}_{n}\right),
$$

so that $\mathbf{x}_{1} \wedge \mathbf{x}_{2} \wedge \cdots \wedge \mathbf{x}_{n}$ is pseudo orthogonal to any $\mathbf{x}_{i}(i=1, \ldots, n)$.

\section{Principal configurations on spacelike submanifolds of codimension two}

Let $\mathbb{R}_{1}^{n+1}$ be an oriented and timelike oriented space and choose $\mathbf{e}_{0}=(1,0, \ldots, 0)$ as the future timelike vector field. Given a spacelike embedding $\mathbf{X}: U \longrightarrow \mathbb{R}_{1}^{n+1}$ from an open subset $U \subset \mathbb{R}^{n-1}$, we write $M=\mathbf{X}(U)$ and identify $M$ and $U$ through the embedding $\mathbf{X}$, where we say that $\mathbf{X}$ is spacelike if $X_{u_{i}}(i=1, \ldots, n-1)$ are spacelike vectors all over $M$. Therefore, the tangent space $T_{p} M$ of $M$ is a spacelike subspace (i.e., consists of spacelike vectors) for any point $p \in M$. In this case, the pseudo-normal space $N_{p} M$ is a timelike plane (i.e., Lorentz plane) (cf. [31]). We denote by $N(M)$ the pseudonormal bundle over $M$. Let $\mathbf{n}$ be a normal field on $M$. Under the identification of $M$ and 
$U$ through $\mathbf{X}$, we have a linear mapping provided by its derivative, $d_{p}(\mathbf{n}): T_{p} M \longrightarrow$ $T_{p} \mathbb{R}_{1}^{n+1}=T_{p} M \oplus N_{p}(M)$ at each point $p \in M$. By composing this with the orthogonal projections, $\pi^{t}: T_{p} M \oplus N_{p}(M) \rightarrow T_{p}(M)$ and $\pi^{n}: T_{p}(M) \oplus N_{p}(M) \rightarrow N_{p}(M)$, we obtain the $\mathbf{n}$-shape operator

$$
S_{p}(\mathbf{n})=d_{p}(\mathbf{n})^{t}=\pi^{t} \circ d_{p}(\mathbf{n})
$$

and the normal connection with respect to $\mathbf{n}$,

$$
d_{p}(\mathbf{n})^{n}=\pi^{n} \circ d_{p}(\mathbf{n}),
$$

evaluated at the point $p$. Its eigenvectors are called $\mathbf{n}$-principal directions and the corresponding eigenvalues the $\mathbf{n}$-principal curvatures $\{\kappa(\mathbf{n})\}_{i=1}^{n-1}$. The function $K(\mathbf{n})(p)=$ $\operatorname{det} S_{p}(\mathbf{n})$ is called Gauss-Kronecker $\mathbf{n}$-curvature. The points at which $K(\mathbf{n})$ vanishes are called $\mathbf{n}$-parabolic points. We say that $\mathbf{n}$ is non-degenerate at $p$ provided $K(\mathbf{n})(p) \neq$ 0 , that is, the subset of $\mathbf{n}$-parabolic points is empty. A point at which all the $\mathbf{n}$-principal curvatures coincide is said to be a $\mathbf{n}$-umbilic. We say that a normal field $\mathbf{n}$ is umbilic over $M$, or alternatively, that manifold $M$ is $\mathbf{n}$-umbilical, if all the points of $M$ are $\mathbf{n}$-umbilic.

Since $N(M)$ is a trivial bundle, we can arbitrarily choose a future directed unit timelike normal section $\mathbf{n}^{T}(u) \in N_{p}(M)$, where $p=\mathbf{X}(u)$. Here, we say that $\mathbf{n}^{T}$ is future directed if $\left\langle\mathbf{n}^{T}, \mathbf{e}_{0}\right\rangle<0$. Therefore we can construct a spacelike unit normal section $\mathbf{n}^{S}(u) \in N_{p}(M)$ by

$$
\mathbf{n}^{S}(u)=\frac{\mathbf{n}^{T}(u) \wedge \mathbf{X}_{u_{1}}(u) \wedge \cdots \wedge \mathbf{X}_{u_{n-1}}(u)}{\left\|\mathbf{n}^{T}(u) \wedge \mathbf{X}_{u_{1}}(u) \wedge \cdots \wedge \mathbf{X}_{u_{n-1}}(u)\right\|}
$$

and we have $\left\langle\mathbf{n}^{T}, \mathbf{n}^{T}\right\rangle=-1,\left\langle\mathbf{n}^{T}, \mathbf{n}^{S}\right\rangle=0,\left\langle\mathbf{n}^{S}, \mathbf{n}^{S}\right\rangle=1$. Although we could also choose $-\mathbf{n}^{S}(u)$ as a spacelike unit normal section with the above properties, we fix the direction $\mathbf{n}^{S}(u)$ throughout this paper. We call $\left(\mathbf{n}^{T}, \mathbf{n}^{S}\right)$ a future directed normal frame along $M=\mathbf{X}(U)$. Clearly, the vector $\mathbf{n}^{T}(u) \pm \mathbf{n}^{S}(u)$ is lightlike. Here we choose $\mathbf{n}^{T}+\mathbf{n}^{S}$ as a lightlike normal vector field along $M$. Since $\left\{\mathbf{X}_{u_{1}}(u), \ldots, \mathbf{X}_{u_{n-1}}(u)\right\}$ is a basis of $T_{p} M$, the system $\left\{\mathbf{n}^{T}(u), \mathbf{n}^{S}(u), \mathbf{X}_{u_{1}}(u), \ldots, \mathbf{X}_{u_{n-1}}(u)\right\}$ provides a basis for $T_{p} \mathbb{R}_{1}^{n+1}$.

It has been shown in [20] that given two future directed unit timelike normal sections $\mathbf{n}^{T}(u), \overline{\mathbf{n}}^{T}(u) \in N_{p}(M)$, the corresponding lightlike normal sections $\mathbf{n}^{T}(u)+$ $\mathbf{n}^{S}(u), \overline{\mathbf{n}}^{T}(u)+\overline{\mathbf{n}}^{S}(u)$ are parallel.

By applying the above procedure to the lightlike vector field $\mathbf{n}^{T}+\mathbf{n}^{S}$ as in [20], we obtain $\left(\mathbf{n}^{T}, \mathbf{n}^{S}\right)$-shape operator of $M=\mathbf{X}(U)$ at $p=\mathbf{X}(u)$. Its eigenvectors are called lightcone principal directions with respect to $\left(\mathbf{n}^{T}, \mathbf{n}^{S}\right)$ at $p$, and the corresponding eigenvalues, denoted by $\left\{\kappa_{i}\left(\mathbf{n}^{T}, \mathbf{n}^{S}\right)(p)\right\}_{i=1}^{n-1}$, are the lightcone principal curvatures with respect to $\left(\mathbf{n}^{T}, \mathbf{n}^{S}\right)$ at $p$. A point $p=\mathbf{X}(u)$ is a $\left(\mathbf{n}^{T}, \mathbf{n}^{S}\right)$-umbilic point if all the principal curvatures coincide at $p$ and thus $S_{p}\left(\mathbf{n}^{T}, \mathbf{n}^{S}\right)=\kappa\left(\mathbf{n}^{T}, \mathbf{n}^{S}\right)(p) 1_{T_{p_{0}} M}$, for some function $\kappa$. This gives rise to the $\left(\mathbf{n}^{T}, \mathbf{n}^{S}\right)$-lightcone principal configuration on $M$, composed by the foliations determined by the integral lines of the lightcone principal directions fields with respect to $\left(\mathbf{n}^{T}, \mathbf{n}^{S}\right)$ and the sets of $\left(\mathbf{n}^{T}, \mathbf{n}^{S}\right)$-preumbilics and $\left(\mathbf{n}^{T}, \mathbf{n}^{S}\right)$-umbilics. We observe that this configuration does not depend on the choice of the pair $\left(\mathbf{n}^{T}, \mathbf{n}^{S}\right)$ 
and it is preserved by the Lorentz transformations. Lightcone principal directions whose associated lightcone principal curvature vanishes are called lightcone principal asymptotic directions on $M$. We say that $M=\mathbf{X}(U)$ is totally $\left(\mathbf{n}^{T}, \mathbf{n}^{S}\right)$-umbilic if all points on $M$ are $\left(\mathbf{n}^{T}, \mathbf{n}^{S}\right)$-umbilic.

\section{Lightcone Gauss map and principal configurations}

Given a spacelike embedding $\mathbf{X}: U \longrightarrow \mathbb{R}_{1}^{n+1}$ from an open subset $U \subset \mathbb{R}^{n-1}$, and a point $p=\mathbf{X}(u)$, consider a future directed unit timelike normal section $\mathbf{n}^{T}(u) \in N_{p}(M)$ and the corresponding spacelike unit normal section $\mathbf{n}^{S}(u) \in N_{p}(M)$ constructed in the previous section. Since given any other future directed unit timelike normal section $\overline{\mathbf{n}}^{T}(u)$, we have $\left(\widetilde{\mathbf{n}^{T}+\mathbf{n}^{S}}\right)(u)=\left(\widetilde{\mathbf{n}^{T}+\overline{\mathbf{n}}^{S}}\right)(u) \in S_{+}^{n-1}$, it is possible to define a lightcone Gauss map of $M=\mathbf{X}(U)$ as

$$
\begin{aligned}
\widetilde{\mathbb{L}}: U & \longrightarrow S_{+}^{n-1} \\
u & \longmapsto\left(\mathbf{n}^{T+\mathbf{n}^{S}}\right)(u) .
\end{aligned}
$$

This induces a linear mapping $d \widetilde{\mathbb{L}}_{p}: T_{p} M \longrightarrow T_{p} \mathbb{R}_{1}^{n+1}$ under the identification of $U$ and $M$, where $p=\mathbf{X}(u)$.

We call the linear transformation $\widetilde{S}_{p}=-\pi^{t} \circ d \widetilde{\mathbb{L}}_{p}$ the normalized lightcone shape operator of $M=\mathbf{X}(U)$ at $p$. The normalized lightcone Gauss-Kronecker curvature of $M=\mathbf{X}(U)$ is defined to be $\widetilde{K}_{\ell}(u)=\operatorname{det} \widetilde{S}_{p}$. We say that $p=\mathbf{X}(u)$ is a lightlike parabolic point if $\widetilde{K}_{\ell}(u)=0$.

The eigenvalues $\left\{\widetilde{\kappa}_{i}(p)\right\}_{i=1}^{n-1}$ of $\widetilde{S}_{p}$ are called normalized lightcone principal curvatures. It follows from the above formula that $\widetilde{\kappa}_{i}(p)=\left(1 / \ell_{0}\right) \kappa_{i}\left(\mathbf{n}^{T}, \mathbf{n}^{S}\right)(p)$. Clearly, the eigenvectors of $\widetilde{S}_{p}$ coincide with the lightcone principal directions with respect to $\left(\mathbf{n}^{T}, \mathbf{n}^{S}\right)$, for any future directed frame $\left(\mathbf{n}^{T}, \mathbf{n}^{S}\right)$ on $M$, therefore, we can refer to the $\left(\mathbf{n}^{T}, \mathbf{n}^{S}\right)$-lightcone principal configuration, simply as the lightcone principal configuration on $M$. The $\left(\mathbf{n}^{T}, \mathbf{n}^{S}\right)$-umbilics shall be called lightlike umbilics. We say that $M=\mathbf{X}(U)$ is totally lightlike umbilical if all points on $M$ are lightlike umbilic. The point $p$ is called a lightlike flat point if $p$ is both lightlike umbilic and parabolic. The spacelike submanifold $M=\mathbf{X}(U)$ is called lightlike flat provided every point of $M$ is lightlike flat. As observed in the previous section, the lightcone principal configuration is preserved by Lorentz transformations, although the lightcone principal curvatures are not. Nevertheless, we shall see below that the Lorentz transformations preserve the lightcone principal asymptotic directions and hence the lightlike parabolic points and the lightlike flat points. Therefore, the lightlike flatness is a Lorentzian property.

4.1 Particular case: $\boldsymbol{M}=\mathbf{X}(\boldsymbol{U}) \subset \boldsymbol{H}_{+}^{n}(-\mathbf{1})$. We can take $\mathbf{n}^{T}=\mathbf{X}$, then $\mathbf{n}^{S}=$ $\mathbf{e} \in S_{1}^{n}$ is univocally defined, and we have that the lightcone Gauss map on $M$ coincides with the hyperbolic Gauss map ([19]), given by

$$
\widetilde{\mathbb{L}}\left(u_{0}\right)=\mathbf{X}\left(\widetilde{\left.u_{0}\right)+\mathbf{e}}\left(u_{0}\right) .\right.
$$


In this case, we call $\widetilde{S}_{p}$, the horospherical shape operator, the corresponding eigenvectors, eigenvalues and umbilics are respectively called horospherical principal directions, horospherical principal curvatures and horoumbilics and determine the horospherical principal configuration on $M$. We thus have that the horospherical principal directions of $M$ at a point $\mathbf{p}_{0}$, considered as a hypersurface of $H_{+}^{n}(-1)$, coincide with the lightcone principal directions of $M$ at $\mathbf{p}_{0}$, considered as a codimension 2 submanifold of $\mathbb{R}_{1}^{n+1}$.

\section{Height functions and contacts with hyperplanes}

Let $M_{i}, N_{i}(i=1,2)$ be submanifolds of $\mathbb{R}^{n}$ with $\operatorname{dim} M_{1}=\operatorname{dim} M_{2}$ and $\operatorname{dim} N_{1}=$ $\operatorname{dim} N_{2}$. We say that the contact of $M_{1}$ and $N_{1}$ at $y_{1}$ is of the same type as the contact of $M_{2}$ and $N_{2}$ at $y_{2}$ if there is a diffeomorphism germ $\Phi:\left(\mathbb{R}^{n}, y_{1}\right) \longrightarrow\left(\mathbb{R}^{n}, y_{2}\right)$ such that $\Phi\left(M_{1}\right)=M_{2}$ and $\Phi\left(N_{1}\right)=N_{2}$. In this case we write $K\left(M_{1}, N_{1} ; y_{1}\right)=K\left(M_{2}, N_{2} ; y_{2}\right)$. It is clear that in the definition $\mathbb{R}^{n}$ could be replaced by any manifold. In his paper [27], Montaldi gives the following characterization of the notion of contact by using the terminology of singularity theory (see [8] for the definition of $\mathcal{K}$-equivalence):

Theorem 5.1. Let $M_{i}, N_{i}(i=1,2)$ be submanifolds of $\mathbb{R}^{n}$ with $\operatorname{dim} M_{1}=\operatorname{dim} M_{2}$ and $\operatorname{dim} N_{1}=\operatorname{dim} N_{2}$. Let $g_{i}:\left(M_{i}, x_{i}\right) \longrightarrow\left(\mathbb{R}^{n}, y_{i}\right)$ be immersion germs and $f_{i}:\left(\mathbb{R}^{n}, y_{i}\right) \longrightarrow\left(\mathbb{R}^{r}, 0\right)$ be submersion germs with $\left(N_{i}, y_{i}\right)=\left(f_{i}^{-1}(0), y_{i}\right)$. Then $K\left(M_{1}, N_{1} ; y_{1}\right)=K\left(M_{2}, N_{2} ; y_{2}\right)$ if and only if $f_{1} \circ g_{1}$ and $f_{2} \circ g_{2}$ are $\mathcal{K}$-equivalent.

So, given two submanifolds $M$ and $N$ of $\mathbb{R}^{n}$, with a common point $p$, and an immersion germ $g:(M, x) \longrightarrow\left(\mathbb{R}^{n}, p\right)$ and a submersion germ $f:\left(\mathbb{R}^{n}, p\right) \longrightarrow\left(\mathbb{R}^{r}, 0\right)$, such that $N=f^{-1}(0)$, we have that the contact of $M \equiv g(M)$ and $N$ at $p$ is completely determined by the singularity type of the germ $(f \circ g, x)$. When $N$ is a hypersurface, we have $r=1$, and the function germ $(f \circ g, x)$ has a degenerate singularity if and only if its Hessian, $\mathcal{H}(f \circ g)(x)$, is a degenerate quadratic form. In such case, the tangent directions lying in the kernel of this quadratic form are called contact directions for $M$ and $N$ at $p$.

We consider now three families of functions that describe respectively the contacts of $M=\mathbf{X}(U)$ with spacelike, timelike and lightlike hyperplanes in $\mathbb{R}_{1}^{n+1}$.

1. The timelike height functions family, given by

$$
\begin{aligned}
H^{t}: U \times H_{+}^{n}(-1) & \longrightarrow \mathbb{R} \\
(u, \mathbf{v}) & \longmapsto\langle\mathbf{X}(u), \mathbf{v}\rangle .
\end{aligned}
$$

2. The spacelike height functions family, given by

$$
\begin{aligned}
H^{s}: U \times S_{1}^{n} & \longrightarrow \mathbb{R} \\
(u, \mathbf{v}) & \longmapsto\langle\mathbf{X}(u), \mathbf{v}\rangle .
\end{aligned}
$$

3. The lightcone height functions family, given by

$$
\begin{aligned}
H^{\ell}: U \times S_{+}^{n-1} & \longrightarrow \mathbb{R} \\
(u, \mathbf{v}) & \longmapsto\langle\mathbf{X}(u), \mathbf{v}\rangle .
\end{aligned}
$$


We denote the Hessian matrix of the $h_{v_{0}}^{t}(u)=H^{t}\left(u, \mathbf{v}_{0}\right)$ (respectively $h_{v_{0}}^{s}, h_{v_{0}}^{\ell}$ ) at $u_{0}$, as $\operatorname{Hess}\left(h_{v_{0}}^{t}\right)\left(u_{0}\right)$ (respectively $\operatorname{Hess}\left(h_{v_{0}}^{s}\right)\left(u_{0}\right)$, Hess $\left.\left(h_{v_{0}}^{\ell}\right)\left(u_{0}\right)\right)$. A normal direction $v \in N_{p} M$ is said to be binormal provided it induces a degenerate height function on $M$ at $p$. The field $\mathbf{n}$ is said to be a binormal field on $M$ provided $\mathbf{n}(p)$ is a binormal direction at $p$, for all $p \in M$. It is not difficult to check that for an appropriate local coordinates system the matrix of the shape operator $S_{p}(\mathbf{n})$ coincides with that of $\operatorname{Hess}\left(h_{\mathbf{n}(u)}^{t}\right)(u)$. By using similar methods to those used in [25] in the case of codimension 2 submanifolds of Euclidean space, we can show that the number of binormal directions (or of osculating hyperplanes) at any point of $M$ is at most $(n-1)$.

A tangent direction $w$ of $M$ at $p=\mathbf{x}(u)$ is said to be an principal asymptotic direction associated to some timelike (respectively spacelike, lightlike) binormal field $\mathbf{n}$ at $p$ provided $u$ is a degenerate singularity of the height function $h_{\mathbf{n}(u)}^{t}$ (respectively $\left.h_{\mathbf{n}(u)}^{s}, h_{\mathbf{n}(u)}^{\ell}\right)$ and $w$ lies in the kernel of $\operatorname{Hess}\left(h_{\mathbf{n}(u)}^{t}\right)(u)\left(\right.$ respectively $\operatorname{Hess}\left(h_{\mathbf{n}(u)}^{s}\right)(u)$, $\operatorname{Hess}\left(h_{\mathbf{n}(u)}^{\ell}\right)(u)$ ). In other words, $w$ is a contact direction of $M$ with some spacelike (respectively timelike, lightlike) hyperplane at $p$. This hyperplane is said to be an osculating hyperplane. It can be shown that the principal asymptotic directions determine tangent lines with higher order contact with the submanifold at the corresponding points.

We observe that given some globally defined binormal field $\mathbf{b}$ on $M$, we have an associated foliation of principal asymptotic curves (with possible critical points) on $M$. This foliation coincides with one of the principal foliations (with vanishing principal curvature) associated to the normal field $\mathbf{b}$ on $M$.

Proposition 5.2 ([20, Proposition 4.2]).

(1) $\partial H^{\ell} / \partial u_{i}\left(u_{0}, \mathbf{v}_{0}\right)=0(i=1, \ldots, n-1)$ if and only if $\mathbf{v}_{0}=\widetilde{\mathbb{L}^{ \pm}}\left(u_{0}\right)$, where $\mathbb{L}^{ \pm}(u)=$ $\mathbf{n}^{T}(u) \pm \mathbf{n}^{S}(u)$.

Moreover, in an appropriate coordinate system, $\operatorname{Hess}\left(h_{v_{0}}^{\ell}\right)\left(u_{0}\right)=\widetilde{S}_{p_{0}}$, and we have

(2) $p_{0}$ is a lightlike parabolic point if and only if $\operatorname{det} \operatorname{Hess}\left(h_{v_{0}}^{\ell}\right)\left(u_{0}\right)=0$.

(3) $p_{0}$ is a lightlike flat point if and only if $\operatorname{rank} \operatorname{Hess}\left(h_{v_{0}}^{\ell}\right)\left(u_{0}\right)=0$.

5.1 Particular case: $\boldsymbol{M}=\mathbf{X}(\boldsymbol{U}) \subset \boldsymbol{H}_{+}^{n}(-\mathbf{1})$. Given a vector $\mathbf{v} \in H_{+}^{n}(-1)$ (respectively $S_{1}^{n}, S_{+}^{n-1}$ ) and a real number $c$, denote by $S(\mathbf{v}, c)$ the hypersphere (respectively equidistant hypersurface, hyperhorosphere) determined by the intersection of the hyperplane $H P(\mathbf{v}, c)$ with $H_{+}^{n}(-1)$. Given $p=\mathbf{X}(u) \in M$, suppose that $\mathbf{v} \in N_{p} M$.

Lemma 5.3. The germ of the height function $h_{\mathbf{v}}^{t}$ (respectively $h_{\mathbf{v}}^{s}, h_{\mathbf{v}}^{\ell}$ ) at $p$ describes the contact of $M=\mathbf{X}(U)$ and the hypersphere (respectively equidistant hypersurface, hyperhorosphere) $S(\mathbf{v}, c)=\left\{\mathbf{x} \in H_{+}^{n}(-1) \mid\langle\mathbf{v}, \mathbf{x}\rangle=c\right\}$ at $p$, where $\langle\mathbf{v}, p\rangle=c$.

Proof. For any $\mathbf{v} \in \mathbb{R}^{n+1}$, consider the function $\lambda_{\mathbf{v}, c}: \mathbb{R}^{n+1} \rightarrow \mathbb{R}$ given by $\lambda_{\mathbf{v}, c}(x)=$ $\langle\mathbf{x}, \mathbf{v}\rangle-c$. Denote by $\bar{\lambda}_{\mathbf{v}, c}$ the restriction of $\lambda_{\mathbf{v}, c}$ to $H_{+}^{n}(-1)$. So $\bar{\lambda}_{\mathbf{v}, c}^{-1}(0)$ is respectively a hypersphere, equidistant hypersurface, or hyperhorosphere in $H_{+}^{n}(-1)$, according to $\mathbf{v}$ is timelike, spacelike, or lightlike. Clearly, $\lambda_{\mathbf{v}, c} \cdot \mathbf{X}=\bar{\lambda}_{\mathbf{v}, c} \cdot \mathbf{X}$ coincides respectively with $h_{\mathbf{v}}^{t}, h_{\mathbf{v}}^{s}$ or $h_{\mathbf{v}}^{\ell}$ and we have the required result. 
Therefore, we have that for $M \subset H_{+}^{n}(-1)$ the singularities of the lightcone height functions family measure the contacts of $M$ with hyperhorospheres in $H_{+}^{n}(-1)$.

Corollary 5.4. The horospherical principal directions of a hypersurface $M$ in $H_{+}^{n}(-1)$ coincide with the principal asymptotic directions determined by the family $H^{\ell}$ of lightlike height functions on $M$.

Proof. The principal asymptotic directions associated to $H^{\ell}$ have been defined as the contact directions corresponding to degenerate singularities of the functions $h_{\mathbf{v}}^{\ell}, \mathbf{v} \in$ $S_{+}^{n-1}$ on $M$. On the other hand, as quoted above, for $\mathbf{v}_{0}=\widetilde{\mathbb{L}}\left(u_{0}\right)$, we can take local coordinates at $u_{0}$ such that $\operatorname{Hess}\left(h_{v_{0}}^{\ell}\right)\left(u_{0}\right)=\widetilde{S}_{p_{0}}$ from which it can be seen that the contact directions corresponding to the degenerate singularities of these functions coincide with the horospherical principal directions too.

The following provide alternative contact function germs for $M$ with hyperspheres, equidistant hypersurfaces and hyperhorospheres in $H_{+}^{n}(-1)$ respectively ([4]):

a) Given $\mathbf{v} \in H_{+}^{n}(-1)$, the distance squared function from $\mathbf{v}, L_{\mathbf{v}}: H_{+}^{n}(-1) \rightarrow \mathbb{R}$, is given by $L_{\mathbf{v}}(\mathbf{x})=\left(\cosh ^{-1}(-\langle\mathbf{x}, \mathbf{v}\rangle)^{2}\right.$. Now, given $p_{0}=\mathbf{X}\left(u_{0}\right) \in M=\mathbf{X}(U)$, let $r \in \mathbb{R}$ be such that $L_{\mathbf{v}}\left(p_{0}\right)=r^{2}$. Then the germ $\left(L_{\mathbf{v}} \cdot \mathbf{X}, u_{0}\right)$ is a contact function germ for the pair $(M, S(\mathbf{v}, r))$ at $p_{0}$, where $S(\mathbf{v}, r)$ denotes the hypersphere $S(\mathbf{v}, r)=$ $H P\left(\mathbf{v}, r^{2}\right) \cap H_{+}^{n}(-1)$.

b) For $\mathbf{v} \in S_{1}^{n}$ and $r \in \mathbb{R}$ such that $L_{\mathbf{v}}\left(p_{0}\right)=r$, we take $L_{\mathbf{v}}: H_{+}^{n}(-1) \rightarrow \mathbb{R}$, given by $L_{\mathbf{v}, r}(\mathbf{x})=\left(\sinh ^{-1}(-\langle\mathbf{x}, \mathbf{v}\rangle)\right.$. Then, analogously, $\left(L_{\mathbf{v}} \cdot \mathbf{X}, u_{0}\right)$ is a contact function germ for $M$ and the equidistant hypersurface given by the intersection $H P(\mathbf{v}, r) \cap H_{+}^{n}(-1)$.

c) For $\mathbf{v} \in L C_{+}^{*}$ and $r \in \mathbb{R}$ such that $L_{\mathbf{v}}\left(p_{0}\right)=r$, we put $L_{\mathbf{v}}(\mathbf{x})=\log (-\langle\mathbf{x}, \mathbf{v}\rangle)$ and again $L_{\mathbf{v}} \cdot \mathbf{X}$ is a contact function for $M$ and the hyperhorosphere $H(\mathbf{v})=\{\mathbf{x} \in$ $\left.H_{+}^{n}(-1) \mid \log (-\langle\mathbf{x}, \mathbf{v}\rangle)=r\right\}$.

It follows from Theorem 5.1 that the functions $h_{\mathbf{v}}^{t}, h_{\mathbf{v}}^{s}$ and $h_{\mathbf{v}}^{\ell}$ must be respectively $\mathcal{K}$-equivalent to the distance functions $L_{\mathbf{v}}$ in a), b) and c).

It was shown in [3] that if $M$ is a compact connected smooth $(n-1)$-manifold immersed in $H_{+}^{n}(-1)$, then every Morse function $L_{\mathbf{v}}$ in a) has exactly two critical points if and only if $M$ is embedded as a metric $(n-1)$-sphere. In view of the above considerations we can now state this in terms of hyperbolic height functions as follows:

Corollary 5.5. Suppose that $M$ is a compact connected smooth $(n-1)$-manifold immersed in $H_{+}^{n}(-1)$. Then every non-degenerate timelike height function has exactly two critical points if and only if $M$ is embedded as a metric $(n-1)$-sphere.

On the other hand, a characterization for complete totally umbilic submanifolds of $H_{+}^{n}(-1)$ in terms of the above distance functions was obtained in [4]. In the case of connected, complete hypersurface $M$ in $H_{+}^{n}(-1)$, it tells us that $M$ is embedded as a hypersphere, hyperhorosphere, or equidistant hypersurface if and only if every non-degenerate function $L_{\mathbf{v}}$ of the types a) and b) above has index 0 or $(n-1)$. So we can rephrase this result in terms of height functions in Minkowski space as follows. 
Corollary 5.6. Suppose $M$ is a connected complete smooth hypersurface in $H_{+}^{n}(-1)$. Then every non-degenerate timelike or spacelike height function on $M$ has index 0 or $(n-1)$ if and only if $M$ is embedded as a hypersphere, hyperhorosphere, or equidistant hypersurface.

\section{Spacelike submanifolds with a parallel normal frame}

We say that a normal vector field $\mathbf{n}$ is parallel if $D_{X} \mathbf{n}=0$, for any $X \in T_{p} M$ and any $p \in M$, where $D_{X} \mathbf{n}$ denotes the normal component of the vector $d \mathbf{n}(X) \in T_{p} \mathbb{R}_{1}^{n+1}=$ $T_{p} M \oplus N_{p} M$.

It can be seen [20] that a manifold $M$ admits a parallel normal frame $\left(\mathbf{n}^{T}, \mathbf{n}^{S}\right)$ made of a timelike and a spacelike vector fields if and only if it admits some parallel normal field $\mathbf{n}$ (which may be either lightlike, timelike or spacelike).

The normal curvature of $M$ at $p$ is defined by

$$
\begin{aligned}
R_{p}^{\perp}: T_{p} M \times T_{p} M \times N_{p} M & \longrightarrow N_{p} M \\
(X, Y, \mathbf{n}) & \longmapsto D_{X}\left(D_{Y} \mathbf{n}\right)-D_{Y}\left(D_{X} \mathbf{n}\right)-D_{[X, Y]} \mathbf{n} .
\end{aligned}
$$

We remind that in case that the normal curvature vanishes identically, then $M$ is said to have flat normal bundle.

Lemma 6.1. A spacelike submanifold $M$ admits locally some parallel normal frame in $\mathbb{R}_{1}^{n+1}$ if and only if its normal curvature vanishes identically.

Proof. It is a well-known property for any connection on a fibre bundle that it is flat if and only if it is locally parallelizable (see for instance [24]).

Corollary 6.2. Suppose that $\mathbf{n}^{T}$ is a parallel timelike field over $M$ and let $p \in M$. Then there exists an orthonormal frame $\left\{\mathbf{X}_{1}, \ldots, \mathbf{X}_{n-1}\right\}$ of $T_{p} M$ of common eigenvectors for the shape operators associated to the fields $\mathbf{n}^{T}, \mathbf{n}^{S}$ and $\mathbf{n}^{T}+\mathbf{n}^{S}$, where $\mathbf{n}^{S}$ is obtained from $\mathbf{n}^{T}$ as in Section 3.

Proof. Since $M$ has a parallel normal vector field, the above lemma implies that its normal curvature vanishes identically. We denote by $S_{p}\left(\mathbf{n}^{T}\right)$ and $S_{p}\left(\mathbf{n}^{S}\right)$ the shape operators of the normal vectors $\mathbf{n}^{T}, \mathbf{n}^{S}$. The Ricci Equation (see [31]) implies that

$$
0=\left\langle R_{p}^{\perp}(X, Y) \mathbf{n}^{T}, \mathbf{n}^{S}\right\rangle=\left\langle S_{p}\left(\mathbf{n}^{T}\right)(X), S_{p}\left(\mathbf{n}^{S}\right)(Y)\right\rangle-\left\langle S_{p}\left(\mathbf{n}^{T}\right)(Y), S_{p}\left(\mathbf{n}^{S}\right)(X)\right\rangle,
$$

for any $X, Y \in T_{p} M$ and $p \in M$. But this can be written as

$$
\left\langle S_{p}\left(\mathbf{n}^{S}\right) \circ S_{p}\left(\mathbf{n}^{T}\right)(X), Y\right\rangle=\left\langle Y, S_{p}\left(\mathbf{n}^{T}\right) \circ S_{p}\left(\mathbf{n}^{S}\right)(X)\right\rangle,
$$

for any $X, Y \in T_{p} M$ and $p \in M$. This is equivalent to the fact that the two self-adjoint operators $S_{p}\left(\mathbf{n}^{T}\right)$ and $S_{p}\left(\mathbf{n}^{S}\right)$ commute, which is also equivalent to saying that they can be diagonalized simultaneously. That is, there is an orthonormal frame $\left\{\mathbf{X}_{1}, \ldots, \mathbf{X}_{n-1}\right\}$ of $T_{p} M$ of common eigenvectors for $S_{p}\left(\mathbf{n}^{T}\right)$ and $S_{p}\left(\mathbf{n}^{S}\right)$. Obviously, the vectors of the frame are also eigenvectors of $S_{p}\left(\mathbf{n}^{T}+\mathbf{n}^{S}\right)$. 
Remark 6.3. It follows that the orthonormal frame $\left\{\mathbf{X}_{1}, \ldots, \mathbf{X}_{n-1}\right\}$ provides a basis of principal directions for any normal field $\mathbf{n}$ on $M$.

Given a unit parallel timelike normal field $\mathbf{n}^{T}$ on a spacelike $(n-1)$-submanifold $M=\mathbf{X}(U)$ of $\mathbb{R}_{1}^{n+1}$, we can consider the map $G_{\mathbf{n}^{T}}: U \rightarrow H_{+}^{n}(-1)$, given by $G_{\mathbf{n}^{T}}(u)=$ $\mathbf{n}^{T}(u)$. If $\mathbf{n}^{T}$ is non-degenerate, then $G_{\mathbf{n}^{T}}$ is an immersion. We denote $\bar{M}=G_{\mathbf{n}^{T}}(M)$ and $\bar{p}_{0}=G_{\mathbf{n}^{T}}\left(u_{0}\right)$, where $p_{0}=\mathbf{X}\left(u_{0}\right)$. We observe that, $\widetilde{\mathbb{L}}(p)=\widetilde{\mathbb{L}}\left(G_{\mathbf{n}^{T}}(p)\right)$, for $p \in M$, where the symbol $\widetilde{\mathbb{L}}$ in the left-hand-side denotes the lightcone Gauss map of $M$ as a codimension 2 submanifold of $\mathbb{R}_{1}^{n+1}$, and in the right-hand-side stands for the horospherical Gauss map on $\bar{M}$ as a hypersurface in $H_{+}^{n}(-1)$.

Proposition 6.4. Let $\mathbf{n}^{T}$ be a unit parallel timelike non-degenerate normal field on $M$. Then we have:

a) $T_{\bar{p}} \bar{M}=T_{p} M, N_{\bar{p}} \bar{M}=N_{p} M$ and $d_{p} G_{\mathbf{n}^{T}}: T_{p} M \rightarrow T_{\bar{p}} \bar{M}$ is an isomorphism.

b) The linear map $d_{p} G_{\mathbf{n}^{T}}$ takes lightcone principal directions of $M$ at $p$ into horospherical principal directions of $\bar{M}$ at $\bar{p}, \forall p \in M$.

Proof. a) Given $X \in T_{p} M$, it follows from the Weingarten equation that

$$
d_{p} G_{\mathbf{n}^{T}}(X)=d_{p} \mathbf{n}^{T}(X)=D_{X} \mathbf{n}^{T}-S_{p}\left(\mathbf{n}^{T}\right)(X) .
$$

If $\mathbf{n}^{T}$ is parallel, we have that $d_{p} G_{\mathbf{n}^{T}}(X)=-S_{p}\left(\mathbf{n}^{T}\right)(X) \in T_{p} M$. This shows that $T_{\bar{p}} \bar{M} \subset T_{p} M$ and the equality follows from the fact that $\mathbf{n}^{T}$ is non-degenerate. Obviously, we also have that $N_{\bar{p}} \bar{M}=N_{p} M$ and $d_{p} G_{\mathbf{n}^{T}}: T_{p} M \rightarrow T_{\bar{p}} \bar{M}$ is an isomorphism.

b) We denote by $I I$ and $\overline{I I}$ the second fundamental forms of $M$ and $\bar{M}$ respectively. That is, given $X, Y \in T_{p} M$, we have

$$
I I(X, Y)=\left(d_{p} \tilde{Y}(X)\right)^{\perp}, \quad \overline{I I}(X, Y)=\left(d_{p} \bar{Y}(X)\right)^{\perp},
$$

where $\tilde{Y}, \bar{Y}$ denote local extensions of $Y$ in $M, \bar{M}$ respectively. Assume that $\bar{Y}$ is given in local coordinates by $\bar{Y}=\sum_{i} f_{j} \frac{\partial}{\partial x_{i}}$ for some functions $f_{i}$ locally defined in a neighborhood of $\bar{p}$ in $\bar{M}$. Then we can also consider the induced extension $\tilde{Y}=\sum_{i}\left(f_{j} \circ G_{\mathbf{n}^{T}}\right) \frac{\partial}{\partial x_{i}}$ in $M$. By using these extensions, we obtain

$$
\begin{aligned}
I I(X, Y) & =\left(d_{p} \tilde{Y}(X)\right)^{\perp} \\
& =\left(\sum_{i} X\left(f_{j} \circ G_{\mathbf{n}^{T}}\right) \frac{\partial}{\partial x_{i}}\right)^{\perp}=\left(\sum_{i} d_{p} G_{\mathbf{n}^{T}}(X)\left(f_{i}\right) \frac{\partial}{\partial x_{i}}\right)^{\perp} \\
& =\left(d_{p} \bar{Y}\left(d_{p} G_{\mathbf{n}^{T}}(X)\right)\right)^{\perp}=\overline{I I}\left(d_{p} G_{\mathbf{n}^{T}}(X), Y\right)=-\bar{I} I\left(S_{p}\left(\mathbf{n}^{T}\right)(X), Y\right) .
\end{aligned}
$$

We denote now by $S_{p}(\mathbf{n})$ and $\bar{S}_{p}(\mathbf{n})$ the shape operators associated to a normal vector $\mathbf{n}$ in $M$ and $\bar{M}$ respectively. The above computation gives us the relationship between both operators:

$$
\bar{S}_{p}(\mathbf{n})=-S_{p}(\mathbf{n}) \circ S_{p}\left(\mathbf{n}^{T}\right)^{-1} .
$$


By Corollary 6.2 there is an orthonormal frame $\left\{\mathbf{X}_{1}, \ldots, \mathbf{X}_{n-1}\right\}$ of $T_{p} M$ of principal directions for the fields $\mathbf{n}^{T}, \mathbf{n}^{S}$ and $\mathbf{n}^{T}+\mathbf{n}^{S}$ in $M$. This means that

$$
S_{p}\left(\mathbf{n}^{T}\right)\left(\mathbf{X}_{i}\right)=\lambda_{i} \mathbf{X}_{i}, \quad S_{p}\left(\mathbf{n}^{S}\right)\left(\mathbf{X}_{i}\right)=\mu_{i} \mathbf{X}_{i},
$$

for some $\lambda_{i} \neq 0$ and $\mu_{i}, i=1, \ldots, n-1$. By using (1), this gives in $\bar{M}$ that

$$
\bar{S}_{p}\left(\mathbf{n}^{T}\right)\left(\mathbf{X}_{i}\right)=-\mathbf{X}_{i}, \quad \bar{S}_{p}\left(\mathbf{n}^{S}\right)\left(\mathbf{X}_{i}\right)=-\frac{\mu_{i}}{\lambda_{i}} \mathbf{X}_{i}
$$

In particular, $\left\{\mathbf{X}_{1}, \ldots, \mathbf{X}_{n-1}\right\}$ are also principal directions for $\mathbf{n}^{T}, \mathbf{n}^{S}$ and $\mathbf{n}^{T}+\mathbf{n}^{S}$ in $\bar{M}$. Note that since $\bar{M}$ is contained in $H_{+}^{n}(-1)$, the lightcone and horospherical principal directions coincide.

Proposition 6.5. Let $\mathbf{n}^{T}$ be a non-degenerate timelike parallel normal field on $M$ and let $H P\left(\mathbf{v}, p_{0}\right)$ represent the hyperplane orthogonal to $\mathbf{v}$ through the point $p_{0} \in M$. Then the contact function of $M$ with $H P\left(\mathbf{v}, p_{0}\right)$ at $p_{0}$ has the same corank and codimension as the contact function of $\bar{M}$ with $H P\left(\mathbf{v}, \bar{p}_{0}\right)$ at $\bar{p}_{0}$. In particular, $M$ has non-degenerate contact with $H P\left(\mathbf{v}, p_{0}\right)$ at $p_{0}$ if and only if $\bar{M}$ has non-degenerate contact with $H P\left(\mathbf{v}, \bar{p}_{0}\right)$ at $\bar{p}_{0}$.

Proof. Assume that $M$ is parameterized locally as $M=\mathbf{X}(U)$, where $u_{0} \in U$ and $p_{0}=\mathbf{X}\left(u_{0}\right)$. The contact function of $M$ with $H P\left(\mathbf{v}, p_{0}\right)$ at $p_{0}$ is denoted by $h_{\mathbf{v}}: U \rightarrow \mathbb{R}$ and is given by $h_{\mathbf{v}}(u)=\langle\mathbf{v}, \mathbf{X}(u)\rangle$.

Analogously, $\bar{M}$ is parameterized locally as $\bar{M}=\mathbb{G}_{\mathbf{n}^{T}} \circ \mathbf{X}(U)$ with $\bar{p}_{0}=\mathbb{G}_{\mathbf{n}^{T}} \circ$ $\mathbf{X}\left(u_{0}\right)$. The contact function of $\bar{M}$ with $H P\left(\mathbf{v}, \bar{p}_{0}\right)$ at $\bar{p}_{0}$ is $\bar{h}_{\mathbf{v}}: U \rightarrow \mathbb{R}$, defined by $\bar{h}_{\mathbf{v}}(u)=\left\langle\mathbf{v}, G_{\mathbf{n}^{T}} \circ \mathbf{X}(u)\right\rangle$.

Because of Proposition 6.4, part a), we deduce

$$
d_{p} G_{\mathbf{n}^{T}}\left(\frac{\partial X}{\partial u_{i}}\right)=\sum_{i=1}^{n-1} a_{i j} \frac{\partial X}{\partial u_{j}},
$$

for some smooth functions $a_{i j}$ such that $\operatorname{det}\left(a_{i j}\right) \neq 0$. From this we obtain that

$$
\frac{\partial \bar{h}_{\mathbf{v}}}{\partial u_{i}}=\sum_{i=1}^{n-1} a_{i j} \frac{\partial h_{\mathbf{v}}}{\partial u_{j}}
$$

In particular, $\bar{h}_{\mathbf{v}}$ and $h_{\mathbf{v}}$ have the same jacobian ideals (i.e., the ideals generated by partial derivatives) in the local algebra $C^{\infty}\left(U, u_{0}\right)$. Since both the corank and the codimension are computed from the jacobian ideals, we deduce that they have the same corank and codimension at $u_{0}$.

In general the contact classes $K\left(M, H P\left(\mathbf{v}, p_{0}\right) ; p_{0}\right)$ and $K\left(\bar{M}, H P\left(\mathbf{v}, \bar{p}_{0}\right) ; \bar{p}_{0}\right)$ do not coincide. Nevertheless, both of them have the same Thom-Boardman symbol [8].

Corollary 6.6. Spacelike $(n-1)$-submanifolds with vanishing normal curvature in $\mathbb{R}_{1}^{n+1}$ admit exactly $n-1$ mutually orthogonal principal asymptotic directions at every point, except at the critical points (at which every direction is a principal asymptotic direction). 
Proof. Let $M$ be a $(n-1)$-submanifold with vanishing normal curvature and let $\mathbf{n}$ be a parallel unit timelike field defined in a neighbourhood of a point $p \in M$. Let $\bar{M}=$ $\mathbf{n}(M) \subset H_{+}^{n}(-1)$. It follows from Proposition 6.5 that a direction $\theta \in T_{p} M$ is a principal asymptotic direction for $M$ at $p$ if and only if $\bar{\theta}=d_{p} L_{\mathbf{n}^{T}}(\theta) \in T_{\bar{p}} \bar{M}$, where $\bar{p}=\mathbf{n}(p)$, is a principal asymptotic direction for $\bar{M}$ at $\bar{p}$. This means that there is some (osculating) hyperplane $H(\mathbf{v}, c)$ having a degenerate contact with $\bar{M}$ at $\bar{p}$ along the direction $\bar{\theta}$. But then it follows from Lemma 6.1 that the hypersphere $S(\mathbf{v}, c)=H(\mathbf{v}, c) \cap H_{+}^{n}(-1)$ of $H_{+}^{n}(-1)$ has degenerate contact with $\bar{M}$ at $\bar{p}$ along the direction $\bar{\theta}$ too. So $\bar{\theta}$ can be seen as a principal direction associated to some normal field on $\bar{M}$.

We consider now a conformal map $\varphi: H_{+}^{n}(-1) \rightarrow \mathbb{R}_{0}^{n}=\left\{\mathrm{x} \in \mathbb{R}_{1}^{n+1} \mid x_{0}=0\right\}$ (for instance, a composition of stereographic projections). This is a diffeomorphism taking hyperspheres (where we include the equivariant hypersurfaces and hyperhorospheres as degenerate ones) in $H_{+}^{n}(-1)$ to hyperspheres (including hyperplanes) in $\mathbb{R}_{0}^{n}$ preserving their respective contacts with $\bar{M}$ and $\varphi(\bar{M})$. Consequently, it determines a bijection between their corresponding contact directions. But these are the principal directions of $\varphi(\bar{M})$ and the principal asymptotic directions of $\bar{M}$. Since $\varphi(\bar{M})$ has exactly $n-1$ orthogonal principal directions at each non-umbilic point, so must have $\bar{M}$. Therefore, $M$ also has exactly $n-1$ orthogonal principal asymptotic directions at each non-critical point.

Remark 6.7. Since each foliation of the principal asymptotic configuration on $M$ can be seen as a principal foliation associated to a binormal field on $M$, it follows that when $M$ has vanishing normal curvature the principal asymptotic foliations grid must coincide with the lightcone principal configuration and its critical points coincide with the lightcone umbilics.

A particular case of codimension 2 spacelike submanifolds with a parallel normal field are those that admit some umbilic normal field. First of all we observe that, as a consequence of the Ricci Equation ([31], p. 125), it can be shown (in a similar manner to the Riemannian case) that if $p$ is an umbilic point for some normal field $\mathbf{n}$ then $R_{p}^{\perp}=0$. Moreover, having vanishing normal curvature on $M$ is equivalent to having flat normal bundle, and thus we have that spacelike submanifolds that admit some umbilic field also admit some parallel normal frame.

It was shown in ([16], Theorem 4.3) that given a spacelike $(n-1)$-submanifold $M$ in $\mathbb{R}_{1}^{n+1}$, which is umbilical for some lightlike parallel normal field $\mathbf{n}$ with curvature $\kappa$, then either $M$ is contained in some lightcone (if $\kappa \neq 0$ ), or $M$ lies in a lightlike hyperplane $(\kappa=0)$. Moreover,

i) If a spacelike $(n-1)$-submanifold $M$ is contained in hyperbolic $n$-space, then the position vector field $\mathbf{X}$ is a parallel timelike normal field along $M$ which is umbilic with constant (non-vanishing) curvature on $M$.

ii) If a spacelike $(n-1)$-submanifold $M$ is contained in de Sitter $n$-space, then the position vector field $\mathbf{X}$ is a parallel spacelike normal field along $M$ which is umbilic with constant (non-vanishing) curvature on $M$. Then we have that the vector field

$$
\nu(u)=\frac{\mathbf{X}(u) \wedge \mathbf{X}_{u_{1}}(u) \wedge \cdots \wedge \mathbf{X}_{u_{n-1}}(u)}{\left\|\mathbf{X}(u) \wedge \mathbf{X}_{u_{1}}(u) \wedge \cdots \wedge \mathbf{X}_{u_{n-1}}(u)\right\|}
$$


is a timelike parallel field globally defined on $M$. We call the map $\nu: M \rightarrow H_{+}^{n}(-1)$ timelike Gauss map on $M$. We observe that $\nu$ is non-degenerate if and only if the $\nu$ parabolic set is empty. In other words, if and only if it defines an immersion of $M$ in $H_{+}^{n}(-1)$.

iii) If a spacelike $(n-1)$-submanifold $M$ is contained in the lightcone of $\mathbb{R}_{1}^{n+1}$, then the position vector field $\mathbf{X}$ is a parallel lightlike normal field along $M$ which is umbilic with constant (non-vanishing) curvature on $M$.

iv) If a spacelike $(n-1)$-submanifold $M$ is contained in a (spacelike, timelike or lightlike) hyperplane of $\mathbb{R}_{1}^{n+1}$, then the normal vector $\mathbf{v}$ to the hyperplane determines a constant (timelike, spacelike or lightlike) normal field along $M$ which is umbilic with vanishing curvature on $M$.

Proposition 6.8. A spacelike ( $n-1)$-submanifold $M$ of $\mathbb{R}_{1}^{n+1}$ is $\mathbf{n}^{L}$-umbilical, for some lightlike normal field $\mathbf{n}^{L}$, if and only if $M$ is contained in some lightcone $L C_{\lambda}$ (provided $\mathbf{n}^{L}$ is not constant) or in a lightlike hyperplane pseudo-orthogonal to $\mathbf{n}^{L}$ (in case that $\mathbf{n}^{L}$ is a constant field).

Proof. Suppose that $\mathbf{n}^{L}$ is a lightlike normal field such that $M$ is $\mathbf{n}^{L}$-umbilical. Then there exists some parallel field $\mathbf{n}^{T}$, that we can assume timelike over $M$. We can construct as in Section 3 another field $\mathbf{n}^{S}$ which is also parallel over $M$. Then the lightlike field $\mathbf{n}^{T}+\mathbf{n}^{S}$ is also parallel and satisfies that $\mathbf{n}^{T}+\mathbf{n}^{S}=\mu \mathbf{n}^{L}$. Since $\mathbf{n}^{L}$ is umbilic, we have that $\mathbf{n}^{T}+\mathbf{n}^{S}$ is also umbilic. Therefore $M$ admits an umbilic lightlike parallel field, which implies the required result.

\section{Global properties of spacelike submanifolds with parallel normal fields}

We shall apply the following idea to different problems in this section: Given a spacelike submanifold $M$ of codimension 2 with a parallel normal field $\mathbf{n}^{T}$ in $\mathbb{R}_{1}^{n+1}$, we use the map $G_{\mathbf{n}^{T}}$, introduced in Section 7, in order to send $M$ into a hypersurface $\bar{M}$ of $H_{+}^{n}(-1)$ that has the same contacts with the hyperplanes of $\mathbb{R}_{1}^{n+1}$ than $M$. Then we use the inverse of stereographic projection in order to send $\bar{M}$ into a hypersurface of the Euclidean space $\mathbb{R}^{n}$, whose contacts with hyperspheres correspond to the contacts of $M$ with the hyperplanes of $\mathbb{R}_{1}^{n+1}$. In this way we can transport some well-known global results on hypersurfaces in Euclidean space to new global results on codimension 2 submanifolds with vanishing normal curvature in Minkowski space.

7.1 Characterization of metric spheres. We consider first the non-degenerate (Morse) contacts of hypersurfaces with hyperspheres and we get the following characterization of total umbilicity in terms of timelike height functions.

Theorem 7.1. Suppose that $M$ is a compact connected smooth spacelike (n-1)-manifold immersed in $\mathbb{R}_{1}^{n+1}$. Then $M$ is a metric $(n-1)$-sphere contained in a spacelike hyperplane if and only if $M$ has a globally defined non-degenerate parallel normal field and every non-degenerate spacelike and timelike height function has exactly two critical points on $M$. 
Proof. We first observe that a metric $(n-1)$-sphere contained in a spacelike hyperplane in $\mathbb{R}_{1}^{n+1}$ necessarily has a globally defined non-degenerate parallel normal field and satisfies that every non-degenerate timelike height function has exactly two critical points on $M$. Conversely, given a compact connected smooth $(n-1)$-manifold with a non-degenerate parallel normal field in $\mathbb{R}_{1}^{n+1}$, that we can choose to be a timelike field $\mathbf{n}^{T}$, we can apply the Proposition 6.5 and use the map $G_{\mathbf{n}^{T}}$ in order to transport $M$ into a hypersurface $\bar{M}$ of $H_{+}^{n}(-1)$ such that $M$ has non-degenerate contact with $H P\left(\mathbf{v}, p_{0}\right)$ at $p_{0}$ if and only if $\bar{M}$ has non-degenerate contact with $H P\left(\mathbf{v}, \bar{p}_{0}\right)$ at $\bar{p}_{0}$, for $\mathbf{v} \in H_{+}^{n}(-1)$ and $\bar{p}_{0} \in M$. Moreover, we have that for any $\mathbf{v} \in H_{+}^{n}(-1)$, the hyperplane $H P\left(\mathbf{v}, p_{0}\right)$ is tangent to $M$ at $p_{0}$ if and only if $H P\left(\mathbf{v}, p_{0}\right)$ is tangent to $\bar{M}$ at $\bar{p}_{0}$. So $p_{0}$ is a (non-degenerate) critical point of $h_{\mathbf{v}}^{t}$ if and only if $\bar{p}_{0}$ is a (non-degenerate) critical point of $h_{\mathbf{v}}^{t}$. It then follows from Corollary 5.5 that $\bar{M}$ is a metric $(n-1)$-sphere (contained in $H_{+}^{n}(-1)$ ). But this implies that $\bar{M}$ is totally umbilic, i.e. all its tangent directions at every point are horospherical principal directions. Now, as a consequence of Proposition 6.4 we get that $M$ must be totally lightlike umbilic and thus a metric $(n-1)$-sphere.

Observe that the existence of some globally defined non-degenerate parallel normal field on $M$ implies that $M$ has vanishing normal curvature and never vanishing Gaussian curvature. We have the following corollary of the above theorem.

Corollary 7.2. Suppose that $M$ is a compact connected spacelike $(n-1)$-submanifold of $S_{1}^{n}$. Then $M$ is a spacelike $(n-1)$-sphere in $S_{1}^{n}$ if and only if the timelike normal is non-degenerate and every non-degenerate timelike height function has exactly two critical points on $M$. Here a spacelike $(n-1)$-sphere in $S_{1}^{n}$ is defined to be the intersection of a spacelike hyperplane with $S_{1}^{n}$.

7.2 Lightcone configurations and Carathéodory type conjectures on surfaces. We look now to the degenerate contacts in order to relate the lightcone configurations of spacelike codimension 2 submanifolds with vanishing normal curvature in Minkowski space with the principal configurations of submanifolds of codimension 2 in Euclidean space.

Consider the conformal map $\varphi: H_{+}^{n}(-1) \rightarrow \mathbb{R}_{0}^{n}=\left\{\mathrm{x} \in \mathbb{R}_{1}^{n+1} \mid x_{0}=0\right\}$ which is given by the composition of the stereographic projections. Since $\varphi$ is a conformal map, it maps the hyperspheres of $H_{+}^{n}(-1)$ into hyperspheres of $\mathbb{R}_{0}^{n}$. On the other hand $\varphi$ is a diffeomorphism and thus preserves contacts and contact directions. Therefore, given any $(n-1)$-submanifold $M \subset H_{+}^{n}(-1)$ we have that $d \varphi$ takes the contact direction of $M$ with any hypersphere $S$ of $H_{+}^{n}(-1)$ at a point $p \in M$ to the contact directions of $\varphi(M)$ with the hypersphere $\varphi(S)$ at $\varphi(p)$ in $\mathbb{R}^{n}$.

Proposition 7.3. Given $M \subset H_{+}^{n}(-1)$, the conformal map $\varphi: H_{+}^{n}(-1) \rightarrow \mathbb{R}_{0}^{n}$ takes the horospherical configuration of $M$ into the principal configuration of $\varphi(M)$ in $\mathbb{R}_{0}^{n}$.

Proof. This follows from the following facts: a) the map $\varphi$ is a conformal map and hence preserves contacts with hyperspheres taking the principal configuration of any hypersurface $M$ in $H_{+}^{n}(-1)$ into the principal configuration of its image $\varphi(M)$ in $\mathbb{R}_{0}^{n}$; b) the 
horospherical principal directions at each point are principal directions for any normal field on $M$ as it can be deduced from Corollary 6.2.

We consider next the particular case of surfaces with a parallel normal field in Minkowski 4-space. A surface $M \subset H_{+}^{3}(-1)$ shall be called generic provided the height functions families $H^{t}, H^{s}$ and $H^{\ell}$ are generic families of functions in the sense that the germ of $\lambda(\mathbf{X})$ at $(u, v)$ is a versal unfolding of the germ of $\mathbf{x}_{v}=\lambda(\mathbf{X})(-, v)$ at $u$, for all $u \in U$ and for all $v \in H_{+}^{3}(-1), S_{1}^{3}$ or $S_{+}^{2}$. We observe that $\varphi$, being a conformal map, induces local $\mathcal{K}$-equivalences between the families of height functions on $M$ and of distance squared functions on $\varphi(M)$ (see [33]). We observe that this means that the family $\lambda(\varphi \cdot \mathbf{X}): U \times \mathbb{R}^{3} \rightarrow \mathbb{R}$ of squared-distance functions on the surface $\varphi(M) \subset \mathbb{R}^{3}$ is a generic family too.

It is a well-known fact that the umbilic points of generically immersed surfaces in $\mathbb{R}^{3}$ are of Darbouxian type, and thus they have index $\pm \frac{1}{2}$ ([36]). Then it follows:

Theorem 7.4. The horospherical configurations in a neighbourhood of a horoumbilical point in a generic surface $M$ in $H_{+}^{3}(-1)$ are of Darbouxian type $D_{i}, i=1,2,3$. Therefore, the index of the lightcone principal direction fields at a lightlike umbilic point of a generic surface generically immersed in $H_{+}^{3}(-1)$ is $\pm \frac{1}{2}$.

And from this we get, as an immediate consequence of the Poincaré-Hopf formula on the lightcone principal configurations, the following horospherical analog to Feldman's result [5] on the number of umbilic points of generic closed surfaces in Euclidean 3-space:

Corollary 7.5. The number of horoumbilical points of any closed (compact without boundary) surface $M$ generically immersed in $H_{+}^{3}(-1)$ is greater or equal than $2|\chi(M)|$, where $\chi(M)$ denotes the Euler number of $M$.

Consequently any 2-sphere generically immersed in $H_{+}^{3}(-1)$ has at least 4 horoumbilical points.

It follows from Montaldi's genericity theorem [26] that the subset of generic immersions of a given surface $M$ in $\mathbb{R}^{3}$ is residual in the Whitney $C^{\infty}$-topology on the total set of immersions of $M$ in $\mathbb{R}^{3}$. Consequently, the subset of generic immersions of a given surface in $H_{+}^{3}(-1)$ is residual in the Whitney $C^{\infty}$-topology on the total set of immersions of $M$ in $H_{+}^{3}(-1)$ too. In a general (non-necessarily generic) situation, there is a Carathéodory conjecture that states that any 2-sphere immersed in Euclidean 3-space has at least 2 umbilics. We can also use the above arguments in order to assert that Loewner's and Carathéodory's conjectures on umbilic points of surfaces in Euclidean 3-space hold if and only if they hold for surfaces in hyperbolic 3-space. Therefore, as a consequence of the proof of the analytic version of Loewner's conjecture for surfaces in $\mathbb{R}^{3}$, we obtain

Theorem 7.6. The index of the horospherical principal direction fields at a horoumbilical point of an analytic surface in $H_{+}^{3}(-1)$ is at most 1 .

From which the following Carathéodory type result follows. 
Corollary 7.7. Any 2-sphere analytically immersed in $H_{+}^{3}(-1)$ has at least two horoumbilical points.

We can now use the considerations made in Section 6 in order to transport these results to the lightcone configurations and lightlike umbilic points of spacelike surfaces with vanishing normal curvature in Minkowski 4-space.

A spacelike surface in $\mathbb{R}_{1}^{4}$ is said to be semiumbilical if it admits some umbilic field locally defined at each one of its points. This is equivalent to asking that the curvature ellipse degenerates into a segment at every point, moreover the umbilic normal field is pseudo-normal to this segment (see [16]). On the other hand, in the particular case of a spacelike surface immersed in 4-dimensional Minkowski space, the semiumbilicity condition is equivalent to the existence of a globally defined parallel normal field. In particular, given such a surface $M$, we can take this normal field to be a timelike field $\mathbf{n}^{T}$. And by means of the map $G_{\mathbf{n}^{T}}$ we obtain a surface $\bar{M}$ immersed in $H_{+}^{3}(-1)$ whose principal configuration is equivalent to that of $M$. Then, as a consequence of the above results on surfaces in $H_{+}^{3}(-1)$ together with Proposition 6.4 we have

Theorem 7.8. Loewner's and Carathéodory's conjectures on umbilic points of surfaces in Euclidean 3-space hold if and only if they hold for semiumbilical spacelike surfaces in Minkowski 4-space.

We observe that these results have been obtained through the analysis of the contacts of the submanifolds with hyperplanes in Minkowski space. Moreover, we have that affine transformations preserve these contacts and thus they take principal asymptotic configurations into principal asymptotic configurations (but do not respect their orthogonality). So we can conclude that the critical points of the principal asymptotic configuration (inflection points) of any spacelike surface which is affinely equivalent to semiumbilic analytic spacelike surface also satisfy the above properties. On the other hand, we cannot say the same with respect to the lightcone principal configurations, for they are preserved by Lorentz transformations (that also preserve the semiumbilicity property) but not by affine transformations. In view of this, we think that it is relevant to push forward the following more general:

Carathéodory type conjecture for spacelike surfaces in $\mathbb{R}_{1}^{\mathbf{4}}$. Any spacelike 2-sphere immersed in $\mathbb{R}_{1}^{4}$ whose principal asymptotic foliations are globally defined has at least two inflection points.

7.3 4-Flattening theorems for closed spacelike curves in Minkowski 3-space. A vertex of a curve $\alpha$ in the Euclidean plane is an extremum of its curvature function. These points can also be characterized as:

a) Singular points of the evolute (locus of centers of curvature) of $\alpha$;

b) Points at which the contact of $\alpha$ with its osculating circle is of order at least three.

Observe that the osculating circle at a point $\alpha(s)$ is characterized by having contact of order at least two with the curve at $\alpha(s)$. This condition can be paraphrased in terms of singularities of distance functions (as in Section 5) by saying that the distance squared 
function from the center of the circle has a singularity of type $A_{k \geq 2}$ at the point $s$. Moreover, the condition b) above is equivalent to saying that a vertex is a singularity of type $A_{k \geq 3}$ of the distance squared function from the corresponding curvature center of $\alpha$ ([2]).

Given a spacelike curve $\gamma: S^{1} \rightarrow \mathbb{R}_{1}^{3}$ in Minkowski 3-space parameterized by the arc-length parameter $s$, we can take $\mathbf{t}(s)=\gamma^{\prime}(s)$ and define the curvature of $\gamma$ as $\kappa(s)=$ $\left\|\gamma^{\prime \prime}(s)\right\|$. If $\kappa(s) \neq 0$, then the unit principal normal vector $\mathbf{n}(s)$ of $\gamma$ at $s$ is given by $\gamma^{\prime \prime}(s)=\kappa(s) \mathbf{n}(s)$. Provided $\gamma^{\prime \prime}(s)$ neither vanishes, nor is a lightlike vector, we have that $\kappa(s) \neq 0$, in such case we define the binormal vector of $\gamma$ at $s$ as $\mathbf{b}(s)=\mathbf{t}(s) \wedge \mathbf{n}(s)$. If we put $\delta(s)=\langle\mathbf{n}(s), \mathbf{n}(s)\rangle$, we have that $\langle\mathbf{b}(s), \mathbf{b}(s)\rangle=-\delta(s)$. Therefore, $\mathbf{b}(s)$ is spacelike (timelike respectively) if and only if $\mathbf{n}(s)$ is timelike (spacelike respectively). Then the following Frenet-Serre type formulae hold:

$$
\begin{aligned}
\mathbf{t}^{\prime}(s) & =\kappa(s) \mathbf{n}(s), \\
\mathbf{n}^{\prime}(s) & =-\delta(s) \kappa(s) \mathbf{t}(s)-\tau(s) \mathbf{b}(s), \\
\mathbf{b}^{\prime}(s) & =\tau(s) \mathbf{n}(s)
\end{aligned}
$$

where $\tau(s)$ is the torsion of $\gamma$ at $s$ ([22]). Analogously to the case of curves in Euclidean 3 -space, we say that a point $\gamma(s)$ is a flattening of $\gamma$ provided $\tau(s)=0$. It is not difficult to see that, analogously to what happens in the Euclidean case ([2]), a point $\gamma(s)$ is a flattening of $\gamma$ if and only if the height function in the direction $\mathbf{b}(s)$ has a singularity of type $A_{k \geq 3}$. In other words, $\gamma$ has contact of order at least three with its osculating plane.

It follows from Lemma 6.1 that in the particular case of a curve immersed in $H_{+}^{2}(-1)$, a flattening is a point at which the curve has contact of order at least three with some circle, equidistant line, or horocycle according to the vector $\mathbf{b}(s)$ is either timelike, spacelike or lightlike. Such points are also known as geodesic vertices of $\gamma$ as a curve in $H_{+}^{2}(-1)$, that is, zeroes of the geodesic curvature. In fact, for a unit speed curve $\gamma: I \rightarrow H_{+}^{2}(-1)$, we can take $\mathbf{t}(s)=\gamma^{\prime}(s)$ as above and define $\mathbf{e}(s)=\gamma(s) \wedge \mathbf{t}(s)$, so we get a pseudoorthonormal frame $\{\gamma, \mathbf{t}, \mathbf{e}\}$ along $\gamma$, for which the following Frenet-Serre type equations hold ([18]):

$$
\begin{aligned}
\gamma^{\prime}(s) & =\mathbf{t}(s), \\
\mathbf{t}^{\prime}(s) & =-\gamma(s)+\kappa_{g}(s) \mathbf{e}(s), \\
\mathbf{e}^{\prime}(s) & =-\kappa_{g}(s) \mathbf{t}(s),
\end{aligned}
$$

where $\kappa_{g}(s)=\operatorname{det}\left(\gamma(s), \mathbf{t}(s), \mathbf{t}^{\prime}(s)\right)$ is the geodesic curvature function on $\gamma$.

Now, we can write $\mathbf{n}$ and $\mathbf{b}$ in terms of $\gamma$ and $\mathbf{e}$ :

$$
\mathbf{n}=\frac{1}{\sqrt{\left|\kappa_{g}^{2}-1\right|}}\left(\gamma+\kappa_{g} \mathbf{e}\right), \quad \mathbf{b}=\frac{1}{\sqrt{\left|\kappa_{g}^{2}-1\right|}}\left(\kappa_{g} \gamma+\mathbf{e}\right)
$$

Provided $\kappa_{g}(s) \neq 1$ (or equivalently, $\kappa(s)=0$ ), we can distinguish two cases:

a) $\kappa_{g}^{2}(s)>1$, which implies that $\delta(s)=-1$ and thus $\mathbf{b} \in H_{+}^{2}(-1)$, b) $\kappa_{g}^{2}(s)<1$, which implies that $\delta(s)=1$ and thus $\mathbf{b} \in S_{1}^{2}$. 
By derivation in the above expression of $\mathbf{n}$, we obtain the following relations between $\tau, \kappa$ and $\kappa_{g}$ :

$$
\tau(s)=\frac{\kappa_{g}^{\prime}}{1-\kappa_{g}^{2}}, \quad \kappa(s)=\sqrt{\left|\kappa_{g}^{2}-1\right|} .
$$

And thus it follows that provided $\kappa\left(s_{0}\right) \neq 0$, then $\gamma\left(s_{0}\right)$ is a flattening if and only if it is a geodesic vertex.

By considering the stereographic projection $\phi: H_{+}^{2}(-1) \rightarrow \mathbb{R}^{2}$ we see, as in the previous section, that since $\phi$ is a conformal map and preserves contacts with circles (equidistant lines and horocycles considered as a particular case), it must take the vertices of a curve in $H_{+}^{2}(-1)$ onto the vertices of its plane image. It now follows from the 4vertex theorem for curves in the Euclidean plane ([28]) that any closed regular simple curve in $H_{+}^{2}(-1)$ has at least 4 vertices (i.e., flattening points). If we allow the curve to have self-intersections then we can only ensure the existence of two flattening points (for any closed plane curve has a minimum and a maximum of its curvature function). We observe that this is a well-known result. A first proof (that uses different tools) was obtained by C. M. Fulton [6] (some related results can be seen in [13] and [40]).

We now use the techniques developed in Section 7 in order to generalize this to a wider class of spacelike curves in Minkowski 3-space. Suppose that $\gamma$ is a closed spacelike curve that admits some globally defined non-degenerate parallel timelike normal field $\nu$. Then the composition $\nu \cdot \gamma=\bar{\gamma}$ is a closed regular curve in the hyperbolic plane $H_{+}^{2}(-1)$. The curve $\bar{\gamma}$ is simple provided $\nu(s) \neq \nu\left(s^{\prime}\right)$, for $s \neq s^{\prime}$. So as a consequence of Proposition 6.5 we can state

Theorem 7.9. Any closed curve that admits a globally defined non-degenerated parallel timelike normal field $\nu$ has at least two flattening points. If $\nu(s) \neq \nu\left(s^{\prime}\right)$, for $s \neq s^{\prime}$, then the curve has at least 4 flattening points.

We now investigate under which conditions we can ensure the existence of some globally defined non-degenerate timelike parallel normal field along the closed spacelike curve $\gamma$ in $\mathbb{R}_{1}^{3}$. We observe first that any parallel field along $\gamma$ must have constant norm, therefore it is either globally timelike, spacelike, or lightlike. Moreover, the existence of a spacelike parallel normal field implies the existence of a timelike one (just rotate this field a right angle in the normal plane of the curve). Let $\nu$ be a unit normal field along $\gamma$. Then we can write $\nu(s)=\cosh \theta(s) \mathbf{n}(s)-\delta \sinh \theta(s) \mathbf{b}(s)$. We observe that $\langle\mathbf{n}, \mathbf{n}\rangle=\langle\nu, \nu\rangle$.

By derivating and applying the Frenet-Serre equations, we get

$$
\begin{aligned}
\nu^{\prime}(s) & =-\theta^{\prime} \sinh \theta \mathbf{n}+\cosh \theta(-\delta \kappa \mathbf{t}-\tau \mathbf{b})-\delta\left(\theta^{\prime} \cosh \theta \mathbf{b}+\sinh \theta \tau \mathbf{n}\right) \\
& =-\delta \kappa \cosh \theta \mathbf{t}-\left(\delta \sinh \theta \tau+\theta^{\prime} \sinh \theta\right) \mathbf{n}-\left(\delta \theta^{\prime} \cosh \theta+\tau \cosh \theta\right) \mathbf{b} .
\end{aligned}
$$

Therefore we have that

a) $\nu$ is parallel if and only if $\theta^{\prime}=-\delta \tau$;

b) $\nu$ is non-degenerate at $s$ if and only if $\nu(s) \neq \mathbf{b}(s)$ and $\kappa(s) \neq 0$.

This tells us that it is always possible to find a locally defined non-degenerate parallel normal field at each point of $\gamma$. Moreover, it follows that such a field can be globally 
defined provided the total torsion $\int \tau$ of $\gamma$ vanishes. In other words, the existence of a globally defined parallel timelike field $\nu$ taking $\gamma$ into a closed spacelike curve $\gamma^{\nu} \subset$ $H_{+}^{2}(-1)$ is equivalent to the vanishing of the total torsion $\int \tau$ of $\gamma$. On the other hand, $\gamma^{\nu}$ is a regular curve if and only if $\nu$ is non-degenerate, which implies that $\gamma$ must have non-vanishing curvature.

In the particular case of a regular simple spacelike curve $\gamma: S^{1} \rightarrow S_{1}^{2}$ in de Sitter 2 -space we have a natural parallel timelike normal field globally defined. In fact, if $\gamma$ has unit speed, we have that the position vector $\gamma(s)$ determines a parallel spacelike normal field along $\gamma$, and then $\gamma(s) \wedge \gamma^{\prime}(s)$ is also a parallel timelike normal field globally defined on $\gamma$. Similarly to the case of curves in hyperbolic plane, we can put $\mathbf{t}(s)=\gamma^{\prime}(s)$ and $\mathbf{e}(s)=\gamma(s) \wedge \mathbf{t}(s)$. Then we get

$$
\begin{aligned}
\gamma^{\prime}(s) & =\mathbf{t}(s), \\
\mathbf{t}^{\prime}(s) & =-\gamma(s)+\kappa_{g}(s) \mathbf{e}(s), \\
\mathbf{e}^{\prime}(s) & =-\kappa_{g}(s) \mathbf{t}(s),
\end{aligned}
$$

where $\kappa_{g}(s)=\operatorname{det}\left(\gamma(s), \mathbf{t}(s), \mathbf{t}^{\prime}(s)\right)$ is the geodesic curvature function on $\gamma$. We call the map e : $S^{1} \rightarrow H_{+}^{2}(-1)$ timelike Gauss map on $\gamma$.

Proceeding as above, we obtain now:

$$
\tau(s)=\frac{\kappa_{g}^{\prime}}{\kappa_{g}^{2}-1}, \quad \kappa(s)=\sqrt{\left|1-\kappa_{g}^{2}\right|} .
$$

And hence, we again have that $\tau(s)=0$ if and only if $\kappa_{g}^{\prime}(s)=0$ and $\kappa_{g}(s) \neq \pm 1$. Points satisfying $\kappa_{g}^{\prime}(s)=0$ are called geodesic vertices of $\gamma$. We observe that $\kappa_{g}(s)= \pm 1$ if and only if $\kappa(s)=0$.

Lemma 7.10. The image $\bar{\gamma}$ of the timelike Gauss map e on $\gamma$ is an embedded curve in $H_{+}^{2}(-1)$ if and only if $\kappa_{g} \neq 0$.

Proof. Since $\mathbf{e}^{\prime}(s)=-\kappa_{g}(s) \mathbf{t}(s)$, we have that e has a singular point at $s$ if and only if $\kappa_{g}(s)=0$. On the other hand, we can write $\kappa_{g}(s)=\left\langle\gamma^{\prime \prime}, \mathbf{e}\right\rangle$. So, provided there are $s_{1}$ and $s_{2}$ such that $\mathbf{e}\left(s_{1}\right)=\mathbf{e}\left(s_{2}\right)=\mathbf{v}$, the points $s_{1}$ and $s_{2}$ are both critical points of the height function $h_{\mathbf{v}}^{t}$. Since $h_{\mathbf{v}}^{t \prime \prime}\left(s_{i}\right)=\kappa_{g}\left(s_{i}\right) \neq 0$, we have that either one of them is a local maximum and the other a local minimum, or both points are local maxima (or minima). If one of them, say $s_{1}$, is a maximum and the other is a minimum then $\kappa_{g}\left(s_{1}\right)<0$ and $\kappa_{g}\left(s_{2}\right)>0$, so there must exist some $s_{0}$ between $s_{1}$ and $s_{2}$ such that $\kappa_{g}\left(s_{0}\right)=0$. In the other case, there must necessarily be a local minimum (or maximum) $s_{3}$ of $h_{\mathbf{v}}^{t}$ between $s_{1}$ and $s_{2}$, but in this case we would have that $\kappa_{g}\left(s_{i}\right)<0(>0), i=1,2$ and $\kappa_{g}\left(s_{3}\right)>0(<0)$, so again there must exist some $s_{0}$ such that $\kappa_{g}\left(s_{0}\right)=0$ and the proof is completed.

We can thus state the following 4-vertex theorem for closed curves in de Sitter 2space: 
Corollary 7.11. Any regular closed spacelike curve immersed in de Sitter 2-space with non-vanishing curvature as a spacelike curve in $\mathbb{R}_{1}^{3}$ and geodesic curvature functions has at least 4 geodesic vertices (flattening points).

We consider now closed spacelike curves in the 2-dimensional lightcone. Let $\gamma$ : $S^{1} \rightarrow L C^{*}$ be such a curve, that we can assume has unit speed. We denote by $\mathbb{R}_{0}^{2}=\{\mathrm{x} \in$ $\left.\mathbb{R}_{1}^{3} \mid x_{0}=0\right\}$ the Euclidean plane in $\mathbb{R}_{1}^{3}$ and by $\mathbf{r}: S^{1} \rightarrow \mathbb{R}_{0}^{2}$ the orthogonal projection of $\gamma$ onto $\mathbb{R}_{0}^{2}$. Let $\mathbf{N}: S^{1} \rightarrow\left\{\mathbf{x} \in \mathbb{R}_{0}^{2} \mid x_{1}^{2}+x_{2}^{2}=1\right\}$ be the (Euclidean) Gauss map of the curve $\mathbf{r}$ and denote by $\gamma^{\ell}: S^{1} \rightarrow L C^{*}$ the lifting of $\mathbf{N}$ to $L C^{*}$ (so $\pi \circ \gamma^{\ell}=\mathbf{N}$, where $\pi: \mathbb{R}_{1}^{3} \rightarrow \mathbb{R}_{0}^{2}$ is the orthogonal projection). We can explicitly write that

$$
\gamma^{\ell}(s)=\left(\frac{\|\mathbf{r}(s)\|}{(\mathbf{r}(s) \cdot \mathbf{N}(s))^{2}}, \frac{\mathbf{r}(s)-2(\mathbf{r}(s) \cdot \mathbf{N}(s)) \mathbf{N}(s)}{(\mathbf{r}(s) \cdot \mathbf{N}(s))^{2}}\right),
$$

where $\mathbf{a} \cdot \mathbf{b}$ is the canonical Euclidean scalar product (i.e., $\mathbf{a} \cdot \mathbf{b}=\langle\mathbf{a}, \mathbf{b}\rangle \mid \mathbb{R}_{0}^{2}$ ). It can be shown that $\left\langle\gamma, \gamma^{\ell}\right\rangle=-2$ (see [15], or [21] for details on this calculation). Since $\gamma$ lies in $L C^{*}$, we have that the position vector $\gamma$ is a parallel lightlike normal field along $\gamma$ and so is $\gamma^{\ell}$. The timelike Gauss map of $\gamma$ is defined as the map e : $S^{1} \rightarrow H_{+}^{2}(-1)$ given by

$$
\mathbf{e}(s)=\frac{\gamma(s)+\gamma^{\ell}(s)}{2} \text {. }
$$

It follows that $\mathbf{e}$ is a globally defined parallel timelike field on $\gamma$. We take $\mathbf{t}(s)=\gamma^{\prime}(s)$ and define the lightcone curvature on $\gamma$ as the function

$$
\kappa_{\ell}=-\left\langle\gamma^{\ell^{\prime}}, \mathbf{t}\right\rangle
$$

Then we have that

$$
\mathbf{e}^{\prime}(s)=\frac{\gamma^{\prime}(s)+\gamma^{\ell^{\prime}}(s)}{2}=\frac{1+\kappa_{\ell}}{2} \mathbf{t} .
$$

So the timelike Gauss curvature on $\gamma$ is given by $\kappa_{\mathbf{e}}=\left(1+\kappa_{\ell}\right) / 2$ and we get that $\kappa_{\mathbf{e}}=0$ if and only if $\kappa_{\ell}=-1 / 2$. An analogous argument to that of Lemma 8.3 shows that the image of $\mathbf{e}$ is an embedded closed curve if and only if $\kappa_{\ell}$ never vanishes. Therefore, we get the following 4-flattening theorem for closed spacelike curves in the 2-dimensional lightcone:

Corollary 7.12. Any regular closed spacelike curve immersed in the 2-dimensional lightcone with non-vanishing timelike Gauss curvature function has at least 4 flattening points.

In [21] we have defined the notion of the total evolute $T E_{\gamma}$ of $\gamma: S^{1} \longrightarrow L C^{*}$ which is decomposed into $T E_{\gamma}=H E_{\gamma} \cup D E_{\gamma}$, where $H E_{\gamma} \subset H^{2}(-1)$ and $D E_{\gamma} \subset S_{1}^{2}$. We have shown that the singularities of the total evolute is corresponding to the flattening points of $\gamma$. Especially these points are the ordinary cusps for generic spacelike curve $\gamma$. By Corollary 8.13, there are at least 4 cusps on the total evolute for generic spacelike curve $\gamma$, some of them are located in $H^{2}(-1)$ and others are in $S_{1}^{2}$. (See also [18]).

We finally observe that having a flattening point is a stable property in the sense that it is preserved by small enough local perturbations of the curve. So we can say that closed spacelike curves which are close enough in the Whitney $C^{3}$-topology to some of the above ones also have at least 4 flattening points. 
Global properties of codimension two spacelike submanifolds in Minkowski space

\section{References}

[1] V. I. Arnol'd, On the Legendrian Sturm theory of space curves. Funktsional. Anal. i Prilozhen. 32 (1998), 1-7, 95. MR1647816 (99m:58030) Zbl 0973.53505

[2] J. W. Bruce, P. J. Giblin, Curves and singularities. Cambridge Univ. Press 1984. MR774048 (87m:58021) Zbl 0534.58008

[3] T. E. Cecil, A characterization of metric spheres in hyperbolic space by Morse theory. Tôhoku Math. J. (2) 26 (1974), 341-351. MR0367875 (51 \#4117) Zbl 0289.53027

[4] T. E. Cecil, P. J. Ryan, Distance functions and umbilic submanifolds of hyperbolic space. Nagoya Math. J. 74 (1979), 67-75. MR535960 (80i:53025) Zbl 0401.53016

[5] E. A. Feldman, On parabolic and umbilic points of immersed hypersurfaces. Trans. Amer. Math. Soc. 127 (1967), 1-28. MR0206974 (34 \#6790) Zbl 0147.21304

[6] C. M. Fulton, The four-vertex theorem in hyperbolic space. J. Differential Geometry 4 (1970), 255-256. MR0264531 (41 \#9123) Zbl 0194.52602

[7] R. A. Garcia, D. K. H. Mochida, M. D. C. Romero Fuster, M. A. S. Ruas, Inflection points and topology of surfaces in 4-space. Trans. Amer. Math. Soc. 352 (2000), 3029-3043. MR1638242 (2000j:58061) Zbl 0966.58020

[8] M. Golubitsky, V. Guillemin, Stable mappings and their singularities. Springer 1973. MR0341518 (49 \#6269) Zbl 0294.58004

[9] C. Gutierrez, F. Mercuri, F. Sánchez-Bringas, On a conjecture of Carathéodory: analyticity versus smoothness. Experiment. Math. 5 (1996), 33-37. MR1412952 (97g:53004) Zbl 0862.57023

[10] C. Gutierrez, F. Sánchez-Bringas, On a Carathéodory's conjecture on umbilics: representing ovaloids. Rend. Sem. Mat. Univ. Padova 98 (1997), 213-219. MR1492978 (98i:53091) Zbl 0897.53003

[11] C. Gutierrez, F. Sánchez-Bringas, On a Loewner umbilic-index conjecture for surfaces immersed in $\mathbb{R}^{4}$. J. Dynam. Control Systems 4 (1998), 127-136. MR1605354 (98k:53007) Zbl 0942.53009

[12] H. L. Hamburger, Beweis einer Caratheodoryschen Vermutung. I / II / III. Ann. of Math. (2) / Acta Math. / Acta Math. 41 / 73 / 73 (1940 / 1941 / 1941), 63-86 / 175-228 / 229 332. MR0001052 (1,172b) / MR0006480 (3,310a) / MR0006481 (3,310b) Zbl 0023.06902 / Zbl 0024.17601 / Zbl 0025.42404

[13] O. Haupt, Vierscheitelsätze in der ebenen hyperbolischen Geometrie. Geometriae Dedicata 1 (1973), 399-414. MR0343224 (49 \#7968) Zbl 0262.53045

[14] V. V. Ivanov, An analytic conjecture of Carathéodory. Sibirsk. Mat. Zh. 43 (2002), 314-405, ii. MR1902826 (2003c:53008) Zbl 1056.53003

[15] S. Izumiya, Legedrian dualities and spacelike hypersurfaces in the lightcone. Mosc. Math. J. 9 (2009), 325-357.

[16] S. Izumiya, D. Pei, M. d. C. Romero Fuster, Umbilicity of space-like submanifolds of Minkowski space. Proc. Roy. Soc. Edinburgh Sect. A 134 (2004), 375-387. MR2056288 (2005c:53077) Zbl 1065.53049

[17] S. Izumiya, D. Pei, T. Sano, Singularities of hyperbolic Gauss maps. Proc. London Math. Soc. (3) 86 (2003), 485-512. MR1971160 (2004h:58056) Zbl 1041.58017 
[18] S. Izumiya, D. H. Pei, T. Sano, E. Torii, Evolutes of hyperbolic plane curves. Acta Math. Sin. (Engl. Ser.) 20 (2004), 543-550. MR2084718 (2005d:53022) Zbl 1065.53022

[19] S. Izumiya, M. d. C. Romero Fuster, The horospherical Gauss-Bonnet type theorem in hyperbolic space. J. Math. Soc. Japan 58 (2006), 965-984. MR2276176 (2007i:53015) Zbl 1111.53042

[20] S. Izumiya, M. d. C. Romero Fuster, The lightlike flat geometry on spacelike submanifolds of codimension two in Minkowski space. Selecta Math. (N.S.) 13 (2007), 23-55. MR2330586 (2008h:53096) Zbl 1141.53066

[21] S. Izumiya, M. Takahashi, Spacelike parallels and evolutes in Minkowski pseudo-spheres. J. Geom. Phys. 57 (2007), 1569-1600. MR2316715 (2008i:53016) Zbl 1121.53036

[22] S. Izumiya, A. Takiyama, A time-like surface in Minkowski 3-space which contains pseudocircles. Proc. Edinburgh Math. Soc. (2) 40 (1997), 127-136. MR1437817 (98a:53100) Zbl 0876.53013

[23] T. Klotz, On G. Bol's proof of Carathéodory's conjecture. Comm. Pure Appl. Math 12 (1959), 277-311. MR0120602 (22 \#11352) Zbl 0091.34301

[24] S. Kobayashi, K. Nomizu, Foundations of differential geometry. Vol I. Interscience Publ. 1963. MR0152974 (27 \#2945) Zbl 0119.37502

[25] D. K. H. Mochida, M. C. Romero-Fuster, M. A. S. Ruas, Osculating hyperplanes and asymptotic directions of codimension two submanifolds of Euclidean spaces. Geom. Dedicata 77 (1999), 305-315. MR1716680 (2000i:58070) Zbl 0942.58039

[26] J. Montaldi, On generic composites of maps. Bull. London Math. Soc. 23 (1991), 81-85. MR1111540 (92d:58018) Zbl 0733.58001

[27] J. A. Montaldi, On contact between submanifolds. Michigan Math. J. 33 (1986), 195-199. MR837577 (87i:58024) Zbl 0601.53007

[28] S. Mukhopadhyaya, New Methods in the geometry of a plane arc. I. Calcutta Bull. Math. Soc. 1 (1909), 31-37. Zbl 40.0624.01

[29] K. Nomizu, L. Rodríguez, Umbilical submanifolds and Morse functions. Nagoya Math. J. 48 (1972), 197-201. MR0315630 (47 \#4179) Zbl 0246.53050

[30] J. J. Nuño-Ballesteros, Submanifolds with a non-degenerate parallel normal vector field in Euclidean spaces. In: Singularity theory and its applications, volume 43 of Adv. Stud. Pure Math., 311-332, Math. Soc. Japan, Tokyo 2006. MR2325143 (2008k:58088) Zbl 1133.53001

[31] B. O'Neill, Semi-Riemannian geometry, volume 103 of Pure and Applied Mathematics. Academic Press 1983. MR719023 (85f:53002) Zbl 0531.53051

[32] M. C. Romero Fuster, Convexly generic curves in $\mathbb{R}^{3}$. Geom. Dedicata 28 (1988), 7-29. MR965828 (89k:53001) Zbl 0654.53003

[33] M. C. Romero Fuster, Stereographic projections and geometric singularities. Mat. Contemp. 12 (1997), 167-182. MR1634431 (99i:58020) Zbl 0939.58036

[34] V. D. Sedykh, Four vertices of a convex space curve. Bull. London Math. Soc. 26 (1994), 177-180. MR1272305 (95a:51036) Zbl 0807.53002

[35] B. Smyth, F. Xavier, A sharp geometric estimate for the index of an umbilic on a smooth surface. Bull. London Math. Soc. 24 (1992), 176-180. MR1148679 (93b:53004) Zbl 0763.53007

[36] J. Sotomayor, C. Gutierrez, Structurally stable configurations of lines of principal curvature. In: Bifurcation, ergodic theory and applications (Dijon, 1981), volume 98 of Astérisque, 195215, Soc. Math. France, Paris 1982. MR724448 (85h:58006) Zbl 0521.53003 
[37] G. Thorbergsson, M. Umehara, A unified approach to the four vertex theorems. II. In: Differential and symplectic topology of knots and curves, volume 190 of Amer. Math. Soc. Transl. Ser. 2, 229-252, Amer. Math. Soc. 1999. MR1738398 (2001f:53009) Zbl 1068.53004

[38] C. J. Titus, A proof of a conjecture of Loewner and of the conjecture of Caratheodory on umbilic points. Acta Math. 131 (1973), 43-77. MR0415543 (54 \#3628) Zbl 0301.53001

[39] M. Umehara, A unified approach to the four vertex theorems. I. In: Differential and symplectic topology of knots and curves, volume 190 of Amer. Math. Soc. Transl. Ser. 2, 185-228, Amer. Math. Soc. 1999. MR1738397 (2001f:53008) Zbl 1068.53005

[40] B. Wegner, A cyclographic approach to the vertices of plane curves. J. Geom. 50 (1994), 186-201. MR1280639 (95c:51013) Zbl 0807.53003

Received 12 October, 2007; revised 23 February, 2008

S. Izumiya, Department of Mathematics, Hokkaido University, Sapporo 060-0810, Japan Email: izumiya@math.sci.hokudai.ac.jp

J. J. Nuño Ballesteros and M. del Carmen Romero Fuster, Departament de Geometría i Topología, Universitat de València, 46100 Burjassot (València), Spain

Email: nuno@uv.es, carmen.romero@uv.es 